\title{
Low-Reynolds-Number Aerodynamics of a Flapping Rigid Flat Plate
}

\author{
Pat Trizila, - Chang-Kwon Kang,,- Hikaru Aono,,- and Wei Shyy \\ University of Michigan, Ann Arbor, Michigan 48109 \\ and \\ Miguel Visbal \\ U.S. Air Force Research Laboratory, Wright-Patterson Air Force Base, Ohio 45433
}

DOI: $\underline{10.2514 / 1 . J 050827}$

\begin{abstract}
Two- and three-dimensional low-aspect-ratio $(\mathrm{AR}=4)$ hovering airfoil/wing aerodynamics at a low Reynolds number $(R e=100)$ are numerically investigated. Regarding fluid physics, in addition to the well-known leading-edge vortex and wake-capture mechanisms, a persistent jet, induced by the shed vortices in the wake during previous strokes, and tip vortices can significantly influence the lift and power performance. While in classical stationary wing theory the tip vortices are seen as wasted energy, here, they can interact with the leading-edge vortex to contribute to the lift generated without increasing the power requirements. Using surrogate modeling techniques, the two- and three-dimensional time-averaged aerodynamic forces were predicted well over a large range of kinematic motions when compared with the Navier-Stokes solutions. The combined effects of tip vortices, leading-edge vortex, and jet can be manipulated by the choice of kinematics to make a three-dimensional wing aerodynamically better or worse than an infinitely long wing. The environmental sensitivity during hovering for select kinematics is also examined. Different freestream strengths and orientations are imposed, with the impact on vortex generation and wake interaction investigated.
\end{abstract}

\section{Introduction}

$\mathbf{M}$ ICRO air vehicles (MAVs) with a maximal dimension of $15 \mathrm{~cm}$ or less and a flight speed of $10 \mathrm{~m} / \mathrm{s}$ are interests of both military and civilian applications. Equipped with a video camera or a sensor, these vehicles can perform surveillance and reconnaissance, and environmental and biochemical sensing, at remote or otherwise hazardous locations. In addition, from the scaling laws [1], a MAV payload is very limited; sensors, batteries, communications equipment, and means of sustained propulsion will have to compete for precious cargo space only in as much as that they will directly help defined mission statements. Research regarding MAVs is growing, as there are still many open challenges in theory and in practice.

The flapping wing variety of MAVs [ $\underline{1}-\underline{3}]$, of interest in the current study, takes a cue from nature and attempts to mimic the success achieved by insects, birds, and bats [4-6]. The study of flapping wing flyers with all of their intricacies is challenging. However, as summarized in [1-3,7-15], significant progress has been made in both engineering and biological communities.

As summarized by Shyy et al. [1,2], several lift enhancement mechanisms have been identified, including leading-edge vortex (LEV), pitchup rotation, wake capture, and clap and fling. Delayed stall comes about as a LEV [16] forms on the upper surface of the airfoil and attaches, even when the wing achieves a high angle of attack (AOA). This in turn produces a low pressure region on the

Presented as Paper 2008-5914 at the 12th AIAA/ISSMO Multidisciplinary Analysis and Optimization Conference, Victoria, British Columbia, Canada, 10-12 Sept. 2008 and Paper 2010-5081 at the 28th AIAA Applied Aerodynamics Conference, Chicago, IL, 28 June-July 1 2010; received 3 August 2010; revision received 15 November 2010; accepted for publication 16 November 2010. Copyright (C) 2011 by Pat Trizila, Chang-Kwon Kang, Hikaru Aono, Miguel Visbal, and Wei Shyy. Published by the American Institute of Aeronautics and Astronautics, Inc., with permission. Copies of this paper may be made for personal or internal use, on condition that the copier pay the $\$ 10.00$ per-copy fee to the Copyright Clearance Center, Inc., 222 Rosewood Drive, Danvers, MA 01923; include the code 0001-1452/11 and $\$ 10.00$ in correspondence with the CCC.

*Department of Aerospace Engineering.

†Department of Aerospace Engineering; weishyy@umich.edu (Corresponding Author) upper surface, which increases the lift. Wake capture refers to when the airfoil switches direction and encounters the previously shed wake, which can then be taken advantage of if at the proper orientation. An important unsteady mechanism, although not performance enhancing, is that of a vortex-induced jet. This flow feature was seen experimentally by Freymuth [17] but has not been adequately studied. Taira and Colonius [18] studied low aspect ratio, impulsively started flat plates at Reynolds numbers from 300 to 500, and, relevant to the current study, saw that when the aspect ratio was four, the midspan flow behavior in two dimensions was vastly different from the three-dimensional (3-D) cases. That same study also saw a performance decrease induced by the tip vortices, which in flapping wing flight can in fact provide a positive contribution, as will be elaborated on in the present investigation.

A model problem has been chosen to probe the unsteady flight mechanisms, their relationship with the hovering kinematics, and the implications on 3-D aerodynamics. It is generally held that, as the aspect ratio is reduced, a potentially strong spanwise variation in the fluid field results. The issue of wing rotation about a joint, due to the centripetal accelerations and extra degrees of freedom, is not investigated. Despite their simplified nature, the chosen kinematics are directly relevant to future MAVs at this scale, and the impact of the flow physics can be extrapolated to other real or imagined cases.

In this work, we assess both two-dimensional (2-D) and 3-D flapping wing fluid physics at a Reynolds number $(R e)$ of 100 , highlighting the role of the various fluid physics, including LEV, wake capture, tip vortices, induced jet, and their overall aerodynamic implications on lift and power during hovering. The Reynolds number in the current study is relevant to small insects and future MAVs at this scale. Prior studies in the literature, applicable to hovering in this flow regime, include those of Freymuth [17], Taira and Colonius [18], Fry et al. [19], Sane and Dickinson [20], Sun and Tang [21], Wang et al. [22], and Shyy and Liu [23]. As will be shown, despite increased interest in the area of flapping wing flight, there are a number of aspects left unaddressed. Regarding the generalization of ideas presented to higher Reynolds numbers [e.g., $\left.\mathcal{O}\left(10^{3}\right)-\mathcal{O}\left(10^{4}\right)\right]$, it has previously been noted $[1,23]$ that, as the sizing and scaling parameters vary, the flow structures can qualitatively differ between those found for small flyers versus larger flyers. 
The main efforts of the study show the following:

1) Tip vortices in low-Reynolds-number flapping wing flight, depending on the flapping kinematics, may have a significant impact on the aerodynamic loading. As presented by Shyy et al. $[2,24]$ in the form of a letter, with details expanded in the current paper, these tip vortices (counter to classical steady-state theory for finite wings) can positively contribute to the performance of the wing instead of being considered wasted energy. The tip vortices affect the impact of the LEV via modified AOA and the anchoring of the coherent vortex structure to the wing; they also influence the strength of the induced jet, to be described next.

2) The presence of an induced jet, observed in the experimental observations of Freymuth [17], in two dimensions is found to decrease lift during the wing-wake interactions.

3) The role of kinematics in 2-D and 3-D geometries is explored. To establish a systematic framework to probe the interplay between flapping kinematics and aerodynamics (time-averaged lift and power), the surrogate modeling technique is employed to represent their relationship. As will be presented, the surrogate models offer a global perspective between aerodynamics and flapping kinematics, including an assessment of the relative importance of these kinematic parameters. In this fashion, trends can be more clearly observed. For example, the surrogate model reveals that, for a considerable portion of the design space, lift is accentuated while the drag (and, subsequently, the power required) is largely unaffected. The characteristics of 2-D versus 3-D hovering can also be probed with desirable clarity. Using the surrogate model, we can clearly identify regions where a 2-D or 3-D wing perform better aerodynamically or where the effect of the aspect ratio is significant. The tool offers new insight into the performance characteristics and helps seek physical mechanisms responsible for the observed behavior. The surrogate process also provides an advantage over isolated case studies in building a more complete understanding of the context of the conclusions; therefore, the relevance/limitations of comparisons with other studies' conclusions can be better established. In this paper, only translational flapping is considered; the rotational flapping will be investigated in the future. While differing from the hovering found in nature, the canonical model employed elicits many of the relevant unsteady fluid physics and is directly relevant to potential MAVs for which the motions are not constrained to those seen in birds and insects.

4) The implication of a light environmental disturbance is investigated on select wing kinematics as a constant freestream is applied at various orientations.

\section{Methodology}

\section{A. Computational Fluid Dynamics Modeling}

The governing equations are the unsteady Navier-Stokes equations with constant density and viscosity; the incompressible versions are shown in Eqs. (1) and (2), written in indicial form:

$$
\begin{gathered}
\frac{\partial}{\partial x_{j}}\left(u_{j}\right)=0 \\
\frac{\partial}{\partial t}\left(u_{i}\right)+\frac{\partial}{\partial x_{j}}\left(u_{j} u_{i}\right)=-\frac{1}{\rho} \frac{\partial p}{\partial x_{i}}+v \frac{\partial}{\partial x_{j}}\left(\frac{\partial u_{i}}{\partial x_{j}}\right)
\end{gathered}
$$

Here, $u_{i}$ is the velocity vector, $x_{i}$ is the Cartesian position vector, $t$ is time, $\rho$ is density of the fluid, $p$ is pressure, and $v$ is the kinematic viscosity of the fluid. Two codes with vastly different methodologies are used in this study; Loci-STREAM and FDL3-DI and are discussed next.

Loci-STREAM [25], used to produce the Navier-Stokes solutions, is a 3-D unstructured pressure-based finite volume solver. It employs implicit first- or second-order time stepping, treats convection terms using the second-order upwind-type scheme $[\underline{26}, 27]$, pressure, and viscous terms using second-order schemes. The system of equations resulting from the linearized momentum equations are fast to converge [27] and are handled with the symmetric Gauss-Seidel [28] solver, which has relatively low memory requirements. The pressure equation [25] is slower to converge, and it is handled by the PETSc Krylov [28] solvers with Jacobi preconditioning. The Loci framework is, by design, highly parallelizable and can take advantage of multiple processors; see Luke and George [29] for a more detailed discussion on rule-based software.

FDL3-DI is a higher-order finite difference solver. It uses a density-based formulation and handles numerical instabilities associated with the higher-order (sixth or eighth) scheme with filtering techniques. For further discussion, the reader is referred to Visbal and Gaitonde [30,31]. These two computational fluid dynamics (CFD) codes were cross validated, and grid and temporal sensitivities were reported in Trizila et al. [32]. Based on these critical examinations, we have confidence in the fidelity of the methods employed.

The translational and rotational airfoil/grid motions are defined as Eqs. (3) and (4) and shown in Fig. 1:

$$
\begin{gathered}
h(t)=h_{a} \sin (2 \pi f t) \\
\alpha(t)=\alpha_{0}-\alpha_{a} \sin (2 \pi f t+\phi)
\end{gathered}
$$

The wing translates back and forth along the $x$ axis while pitching about the midchord along the $z$ axis. Consequently, lift is in the positive $y$ direction, while drag is in the direction opposite of the time varying velocity. Here, $h(t)$ and $h_{a}$ are the dimensional translational position and plunging amplitude, respectively. The angular orientation, time-averaged angle, and angular amplitude are $\alpha(t)$, $\alpha_{0}\left(90^{\circ}\right.$ for all cases under consideration), and $\alpha_{a}$, respectively. The pitching is about the center of the rigid airfoil. The phase lag between the two motions is $\phi$, and the motion frequency is denoted as $f$, whereas the time is again $t$. The three kinematic parameters (called design variables in surrogate modeling), $h_{a}, \alpha_{a}$, and $\phi$, can be varied independently.

Because of the kinematic constraints, there are only two relevant nondimensional groups in the incompressible case. The plunging amplitude to chord ratio, $2 h_{a} / c$, and the Reynolds number:

$$
R e=\frac{U_{\mathrm{ref}} L_{\mathrm{ref}}}{v}=\frac{\left(2 \pi f h_{a}\right) c}{v}
$$

The reference velocity $U_{\text {ref }}$ in this case is the maximum translational velocity, defined by the flapping frequency $f$ and the plunging amplitude $h_{a}$. Since $R e$ is being held constant, $h_{a}$ and $f$ are not independent. Note that the reduced frequency $k$ is not emphasized here as, for hovering, it contains the same information as the plunging amplitude ratio, whereas if $U_{\text {ref }}$ is instead set equal to zero, the reduced frequency is always infinite:

$$
k_{\text {hovering }}=\frac{2 \pi f L_{\mathrm{ref}}}{2 U_{\mathrm{ref}}}=\frac{2 \pi f c}{2\left(2 \pi f h_{a}\right)}=\frac{c}{2 h_{a}}
$$

Figure 2 shows the grid distribution near the flat plate and the applied boundary conditions over the computational domain. The thickness of the flat plate is $0.02 c$, and the flat plate is rectangular: i.e., there is no variation in the spanwise direction ( $z$ axis). For the finite

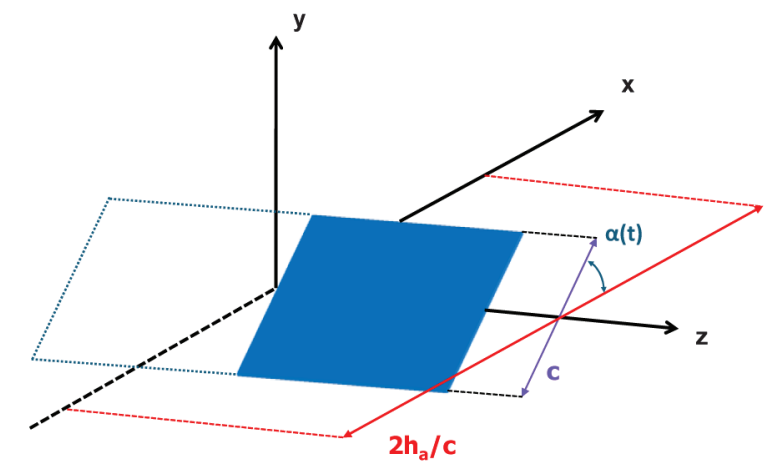

Fig. 1 Illustration of the kinematic parameters for normal hovering. 

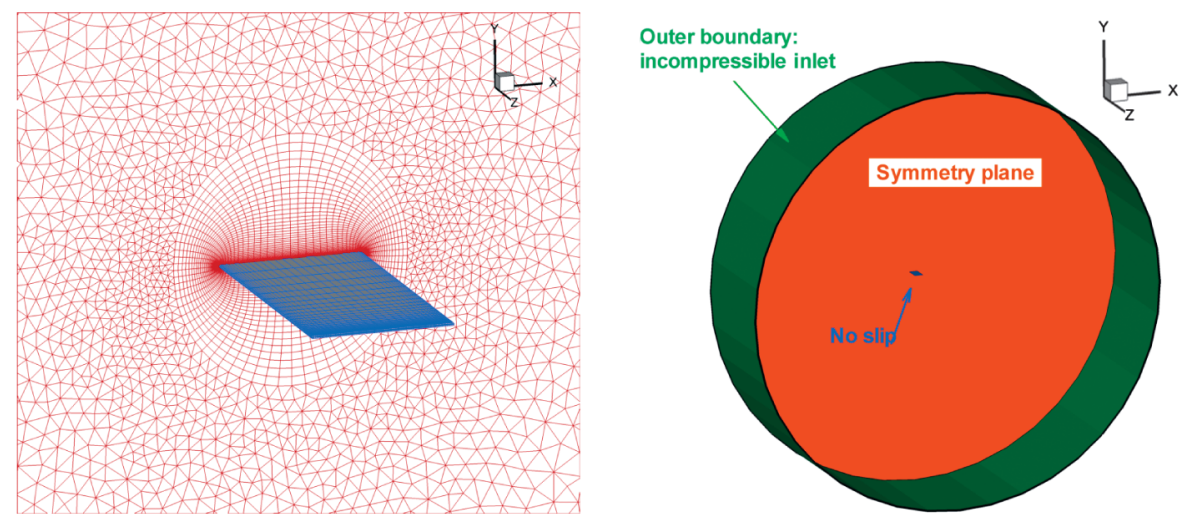

Fig. 2 Computational grid (left) and boundary conditions assigned on the computational domain (right).

wing simulations, $A R=4$. The half-span length from the symmetry plane to the wingtip is $2 c$, the leading edges (LEs) and trailing edges (TEs) are rounded using a half-circular shape, while the wingtip is flat. The fine details of the shape are not seen to have a large influence on the resulting aerodynamics. A more pronounced shape effect is a comparison between a flat plate and a $15 \%$ thickness ellipse (Trizila et al. [33] and Shyy et al. [1]), which saw differences in the magnitude of the force generation but the same qualitative force and flow behavior. The outer boundary is located at $25 c$ away from the flat plate, and the outer boundary plane is opposite to the symmetry plane at $15 c$. At all nonsymmetry plane outer boundaries, inlet conditions with zero velocity conditions are imposed.

\section{B. Surrogate Modeling}

The motivation behind surrogate models is to replace costly objective function evaluations, a quantity of interest such as timeaveraged lift obtained via detailed CFD solutions or substantial experimental setups, with inexpensive approximations of sufficient fidelity, ideally something that could be calculated in real time for use in flight vehicle controllers. Overview of surrogate modeling techniques and selected applications can be found in [34-36]. The process starts with choosing a number and locations of training points in the design space (here meaning the 3-D space encompassing the three kinematic parameters); this is known as constructing the design of experiment (DOE). Once the training points are chosen, the objective functions must be evaluated; depending on the context, this can be done computationally, analytically, and/or experimentally. After the objective functions have been obtained for the selected training points, the surrogate models can be built. Popular models are polynomial response surfaces (PRSs) [37], Kriging [38], radial basis neural networks [39], support vector regression [40] models, and various combinations thereof. After the models are constructed, appropriate error measures can be adopted to construct a weighted-average surrogate (WAS) $[41,42]$. Based on the surrogate, the global sensitivity evaluations can be performed to evaluate the individual and collective influence of the kinematic parameters (design variables) and to order the relative importance of them in determining the aerodynamic outcome.

\section{Design Space}

The range of variables (see Table 1) is comparable to that of a fruit fly and was chosen after considering the length and time scales

Table 1 Minimum and maximum values of the plunging amplitude ratio, angular amplitude, and phase lag evaluated

\begin{tabular}{ccc}
\hline \hline Parameter & Minimum & Maximum \\
\hline $2 h_{a} / c$ & 2.0 & 4.0 \\
$\alpha_{a}$ & $45^{\circ}$ & $80^{\circ}$ \\
$\varphi$ & $60^{\circ}$ & $120^{\circ}$ \\
\hline \hline
\end{tabular}

observed in nature and compiled in [43-45]. Tabulated measurements of angular amplitudes and phase lags for a variety of species are not as well documented and are selected in this study by matching the designated scaling parameters. As already stated, in this study, we focus on the Reynolds number of 100 under hovering conditions to limit the scope.

\section{Design of Experiments}

The number and the efficient distribution of the training points to populate the design space is considered thoroughly in the DOE. The DOE used a face-centered cubic design (FCCD) [35] and then Latin hypercube sampling (LHS) [36] to appropriately fill in the remainder of the design space. The reasoning behind this is that a second-order PRS construction has $(N+1)(N+2) / 2$ coefficients, $N$ being the number of variables and, in general, one wants twice this many data points for an initial curve fit, which would try and reconcile the computational cost (fewer training points desired) with the resulting fidelity (more training points desired). A FCCD design provides $2^{N}+2 N+1$ points: $2^{N}$ corner points, $2 N$ face points, and one center point. Thus, for three design variables, FCCD provides 15 of the 20 points required. The LHS then provides a method for efficiently choosing the rest of the points by maximizing the distance between the added points. A tabulation of the 2-D and 3-D simulations run, and their respective outcomes, as well as quantitative measures of merit for the surrogate models, are found in [33].

\section{Global Sensitivity Analysis}

The global sensitivity analysis (GSA) is, in general, useful for 1) determining if a variable is particularly influential in the design space; if not, perhaps the variable can be fixed and the degrees of freedom and complexity of the problem reduced; 2) ranking the importance of the design variables; and 3) quantifying the degree of coupling between design variables. For example, is the influence on the design space mostly an individual effort, or is there an effect caused by the interaction of variables? For more details, refer to Sobol's method in [36].

\section{Results}

In this section, we use the surrogate modeling technique, using the WAS model $[41,42]$ to establish the relationship between lift generated and power required as the three flapping kinematic parameters vary. The purpose is to gain a global perspective of a multidimensional design space. The utility of the resulting surrogate model is a strong function of what was chosen as the quantity of interest to train it. The current study uses a time-averaged value (e.g., lift) and cannot directly answer questions such as why the lift is high/ low. To gain a more complete picture, the surrogate models are combined with the instantaneous force histories and flowfield visualizations from the CFD simulations. 


\section{A. Time-Averaged Lift}

Figure 3 shows the surrogate models of the time-averaged lift, including those based on the 2-D and 3-D results, as well as their differences. Each axis corresponds to one of the design variables, while the color contours illustrate the objective function of interest (e.g., time-averaged lift or power). Qualitatively, the general trends found in 2-D and 3-D cases are consistent. As the angular amplitude is increased, a lower AOA results, causing decreases of the lift. The general trend for plunging amplitude is also largely consistent between 2-D and 3-D cases. Alter the plunging amplitude, and the resulting lift does not change significantly for much of the design space. There are regions in the design space where the time-averaged lift shows clear differences between 2-D and 3-D cases. A closer inspection of the quantitative relationship between the lift and the kinematic parameters reveals that these differences can be substantial (illustrated in Fig. 3). These will be highlighted shortly.

To show the global impact of the kinematic variables in the entire design space, Fig. 4 illustrates the total variances due to the respective design variables. Immediately apparent is the change in the hierarchy of design variables. In two dimensions, the time-averaged lift was the most sensitive to the angular amplitude, significantly less sensitive to the phase lag, and even less still to the plunging amplitude. While the plunging amplitude was not a negligible influence, such an analysis cannot only rank the relative importance but also illuminate variables that have negligible influence. A finding of this nature can reduce the dimensionality of the design space, greatly reducing the time it takes for refinement iterations, because that variable can effectively be treated as a constant. In this study, the plunging amplitude has been kept as a design variable for completeness.

In the 3-D case, Fig. 4 reveals both the plunging amplitude and the phase lag have substantially increased importance relative to the average lift produced compared with the 2-D case, so much so that the hierarchy of sensitivity changes from 1) $\left.\alpha_{a}, 2\right) \phi$, and 3) $h_{a}$ in 2-D, to 3 -D, where the order is 1) $\phi$, 2) $\alpha_{a}$, and 3) $h_{a}$. In the present context, the main and total variances are close enough to not warrant separate plots. This in turn implies a relatively small degree of coupling
Two-dimensional lift
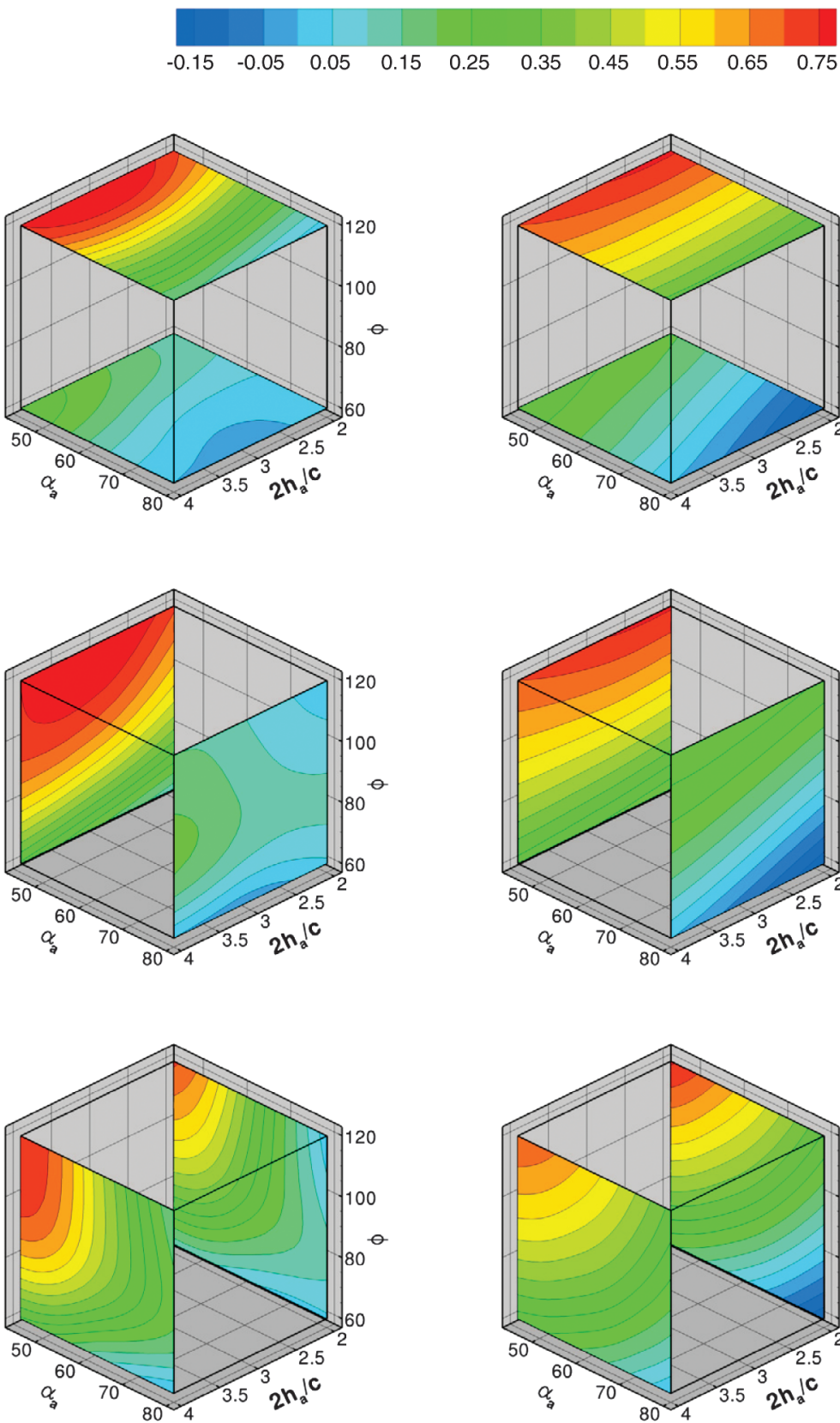

Fig. 3 Surrogate modeling results for lift: 2-D (left), 3-D (middle), and 3-D minus 2-D (right) time-averaged lift.
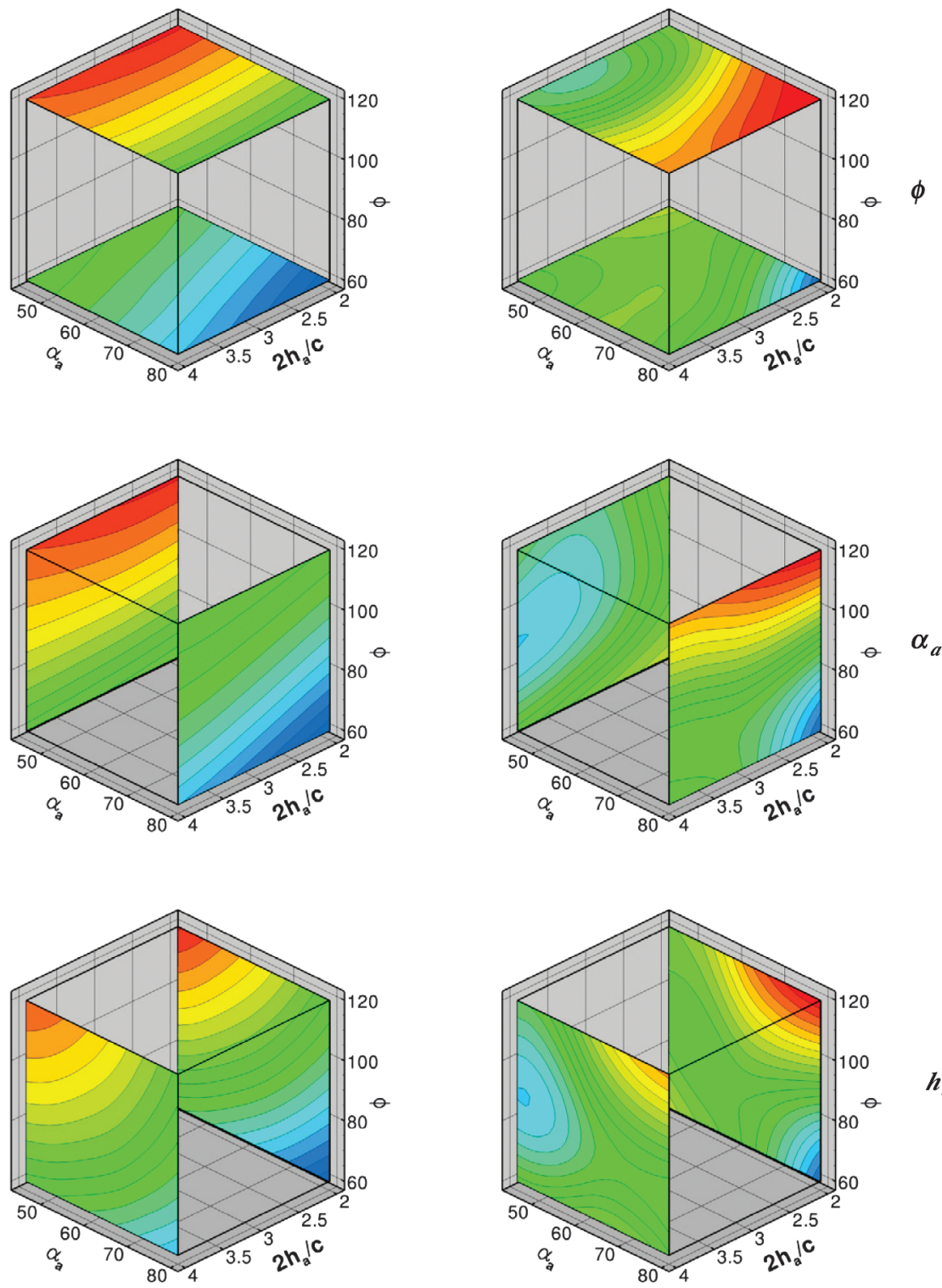

$h_{a}$ 

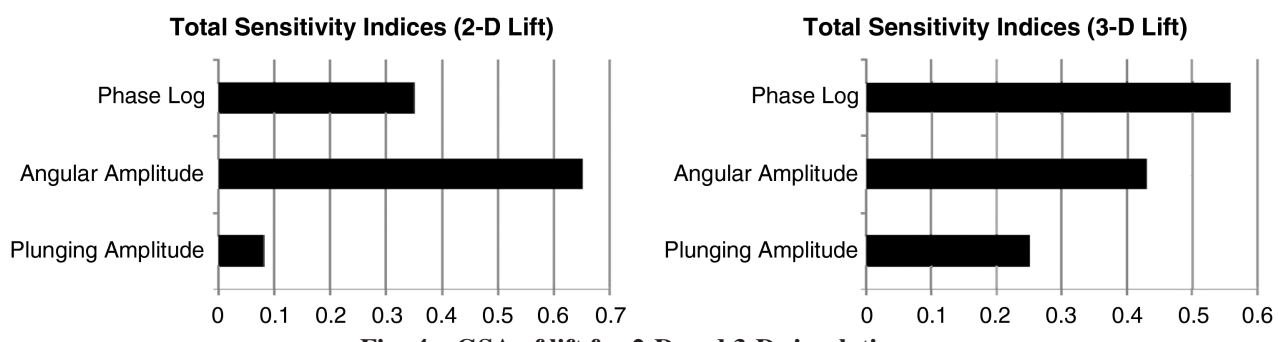

Fig. 4 GSA of lift for 2-D and 3-D simulations.

between the design variables and their resulting effect on the integrated lift.

To identify where in the design space 3-D effects become important, isosurfaces of the absolute difference between the 2-D and 3 -D lift coefficients are shown in Fig. 5 The green regions in the design space correspond to the kinematic parameters with the difference in time-averaged lift coefficient larger than 0.1 due to three-dimensionality. Four coherent regions are identified: region 1, characterized by synchronized hovering and low angular amplitude; region 2, with advanced rotation and high angular amplitude; region 3, with delayed rotation, low angular amplitude, and small plunging amplitude; and region 4, delayed rotation, high angular amplitude, and low plunge amplitude. Everywhere else, the differences between the 2-D and 3-D lift is below the 0.1 threshold. The similarities in this catchall region are defined by the likeness of their time-averaged values but, in some instances, as will be shown, stronger statements may be appropriate.

\section{B. Region 1: Synchronized Hovering, High Angle of Attack}

Region 1 is defined by kinematics that are close to synchronized hovering (i.e., including cases with slight delayed or advanced rotation), low angular amplitude (high AOA), and larger plunging amplitudes. The time history of lift (see Fig. 6) shows two local maxima per stroke in 2-D. The first peak is associated with the wake capture at the beginning of the stroke at $t / T=0.8$, where $t$ is the time that is nondimensionalized by the flapping period $T$. Between the two peaks is a local minimum referred to as a wake valley, which is caused by a combination of decreasing AOA and interaction with a pocket of downward momentum. For this region of the 2-D design space, this pocket of downward momentum takes the form of a persistent jet. As reported during the experimental studies of Freymuth [17], the jet develops as a result of a reverse Karman vortex street interacting with the downward momentum created by the wing as it translates. As the wing passes the jet, vortices are shed with an orientation that reinforces the downward momentum previously

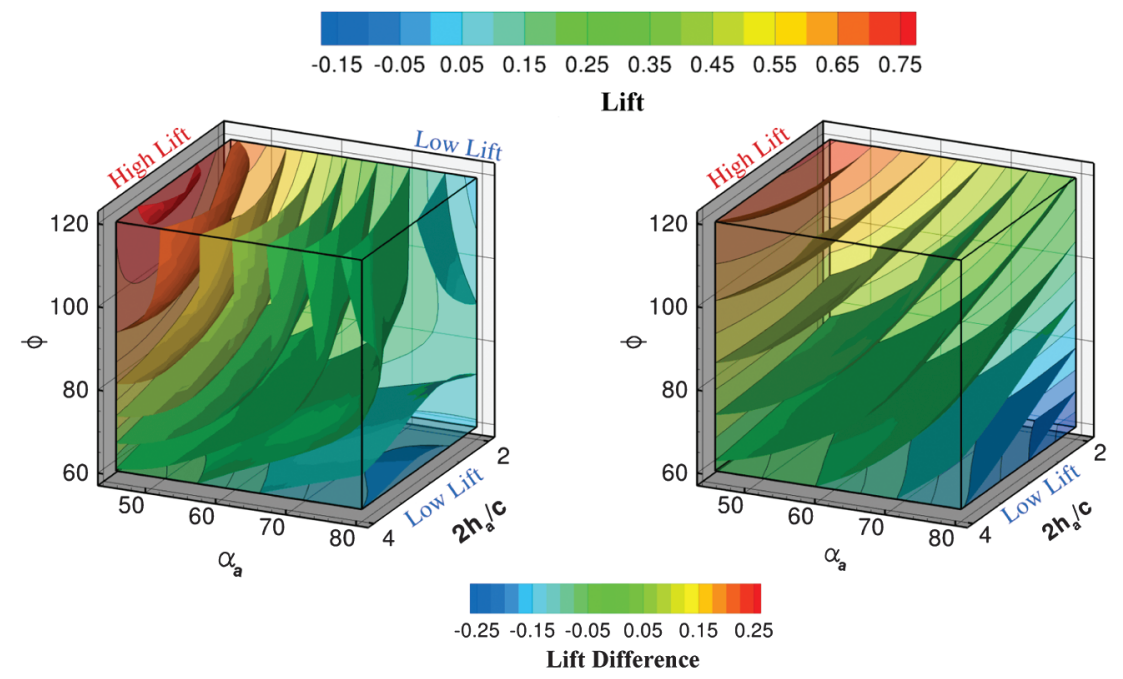

a)

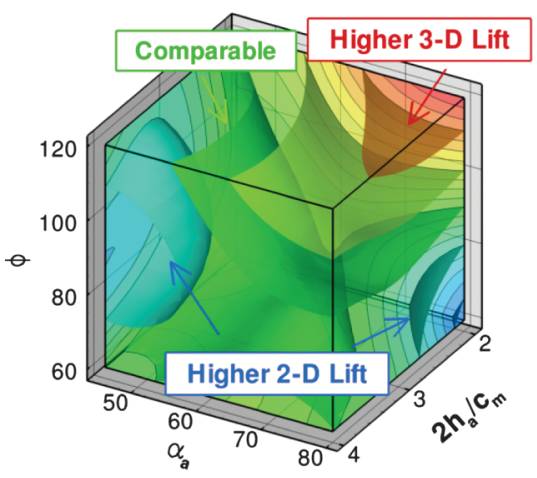

b)

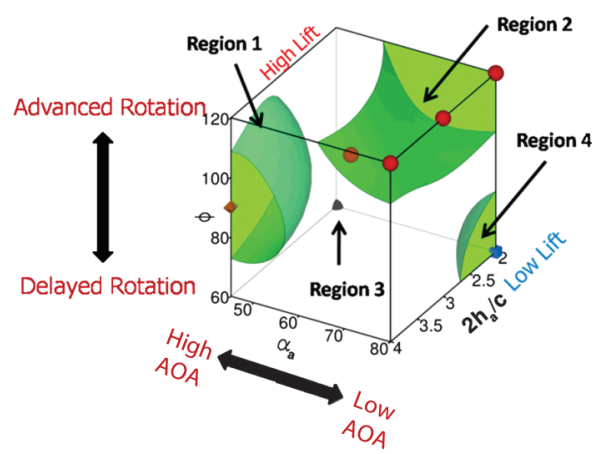

d)

Fig. 5 Surrogate model responses of lift as functions of $2 h_{a} / c, \alpha_{a}$, and $\phi$. 
created by the wing. These vortices sustain the downward momentum, and they further entrain surrounding fluid to create a flow feature that the wing then interacts with during subsequent stroke.

In general, the tip vortices are found to have four prominent competing effects: TE1) enhancement of lift due to the proximity of the associated low pressure region of the tip vortex next to the airfoil; TE2) induced downwash acting to reduce the effective AOA along the span weakening the LEV, hence reducing the instantaneous lift; TE3) interaction with the vortices shed from the LEs and TEs anchoring them from shedding near the wingtips enhancing the lift (region 3); and TE4) because of the tip vortices pulling fluid from the underside of the wing to the upper side, the wing leaves behind a weaker pocket of downward momentum in the flow. Upon interaction with this downward momentum, a loss in lift is seen, and so a weaker wake valley means higher lift.

In the 3-D case, the weaker downward momentum (TE4) pocket does help compared with 2-D; see $t / T=0.9$ in Fig. 6 , which shows the instantaneous lift history (2-D: red; 3-D: black) and vertical velocity contour plots at three time instants in the forward stroke for case $11\left(2 h_{a} / c=3.0, \alpha_{a}=45^{\circ}\right.$, and $\left.\phi=90^{\circ}\right)$ from 2-D computation (Fig. 6a), in the symmetry plane of 3-D computation (Fig. 6b), and near the wingtip $(z / c=1.8)$ (Fig. 6c). In 2-D, the persistent jet shows larger $u_{2}$ magnitudes and is narrower. However, the weaker LEV (TE2) causes the lift to suffer. In the 2-D case, the LEV is largely attached, and the benefit of anchoring the LEV is not
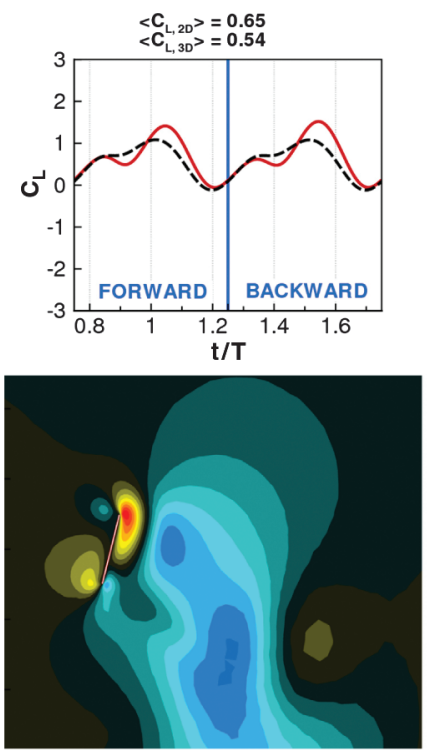

a) 2-D
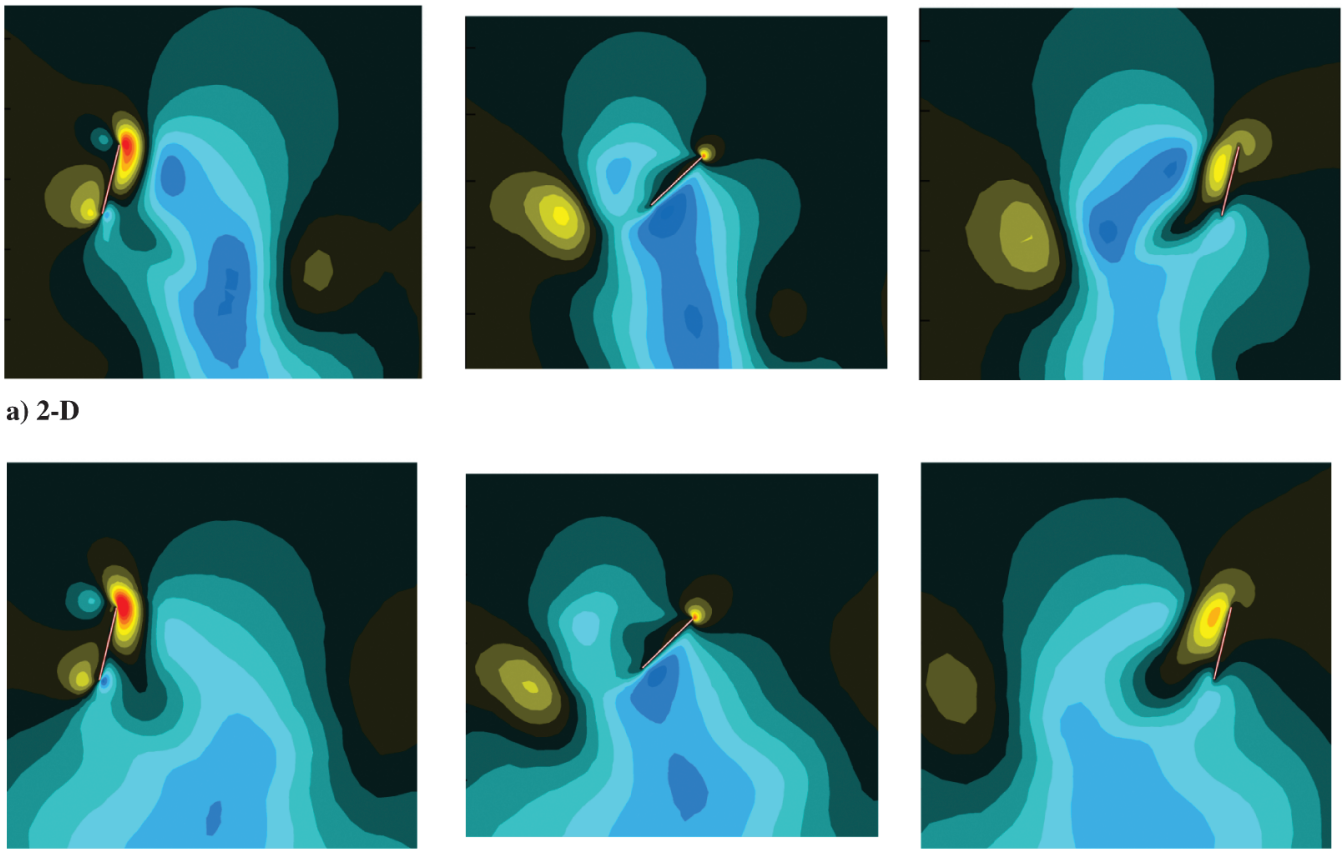

$\mathbf{u}_{2}$ $\begin{array}{lllllllllll}-0.8 & -0.64 & -0.48 & -0.32 & -0.16 & 0 & 0.16 & 0.32 & 0.48 & 0.64 & 0.8\end{array}$

b) 3-D symmetry
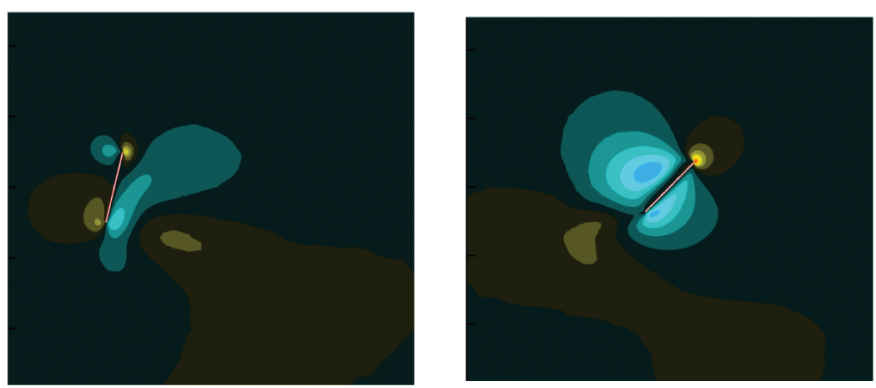

c) 3-D near tip

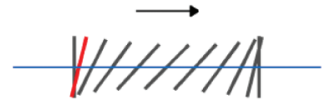

0.8

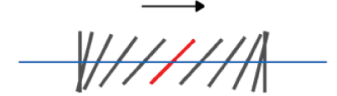

1.0
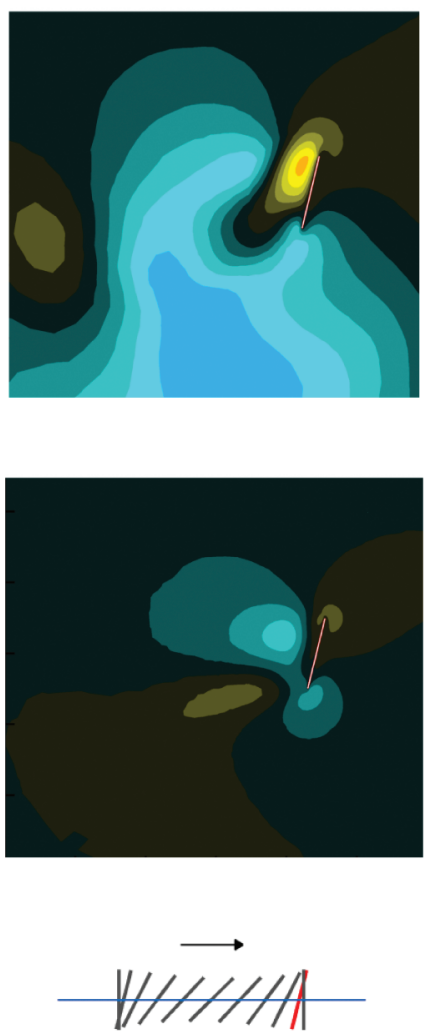

1.2

Fig. 6 Lift history and vertical velocity contours at $t / T=0.8,1.0$, and 1.2 for case 11 . 
helping. The overall impact for this case is that the 2-D lift, $\left\langle C_{L, 2 \mathrm{D}}\right\rangle=0.65$, is better than the $3-\mathrm{D}$ counterpart, $\left\langle C_{L, 3 \mathrm{D}}\right\rangle=0.54$. More generally, cases with kinematics in region 1 have larger lift in 2-D.

\section{Region 2: Advanced Rotation, Low Angle of Attack}

In region 2, the kinematics are characterized by advanced rotation and high angular amplitude.

Figure 7 shows the time histories of lift and the associated flow features from case $8\left(2 h_{a} / c=4.0, \alpha_{a}=80^{\circ}\right.$, and $\left.\phi=120^{\circ}\right)$, which is representative for the other cases in region 2. Right after the stroke reversal, the flat plate moves into the wake generated in the previous stroke. Because of the downwash in this wake (see Fig. 7) and low and decreasing AOA (Fig. 7b), lift drops. Note that the pocket of downward momentum encountered for these kinematics is not a persistent jet. The 3-D case does not suffer the same drop in lift as the 2-D case.

\section{Region 3: Delayed Rotation, High Angle of Attack, Low Plunging Amplitude}

In classical steady-state theory, the presence of wingtip vortices is attributed to a loss of lift and an increase in drag when comparing the 2-D wing to its finite 3-D counterpart [46]. What is seen, in the current study, are instances in which low-aspect-ratio unsteady aerodynamics are producing tip vortices that can enhance lift while negligibly influencing the drag (highlighted in Sec. III.G).

Region 3 is defined by kinematics with delayed rotation, low angular amplitude (high AOA), and shorter plunging amplitudes. This region shows a significant impact from the tip vortices. Figure 8 presents a delayed rotation case with $2 h_{a} / c=2.0, \alpha_{a}=45^{\circ}$, and $\phi=60^{\circ}$. The difference in the flow physics encountered due to 3-D phenomena is noticeable. The main characteristics of the vortices, including sizes, strengths, and movement, are distinctly different between 2-D and 3-D results. Not only is there a strong spanwise variation in the 3-D flow, but there is also little resemblance between the symmetry plane of the 3-D computation and the 2-D computations.

In 2-D flow, the pair of the large-scale vortices are noticeably closer to each other and the airfoil than that in the 3-D flow. The instantaneous lift coefficient for the two cases examined is illustrated in Fig. 9, depicting noticeable differences, in that the 3-D lift coefficient (dashed black) is generally higher than its 2-D counterpart (solid red). With these kinematics patterns, the tip vortices can interact with the LEV to form a lift enhancement mechanism. This aspect will be discussed next.

Figure 9 shows iso- $Q$ [47] surfaces colored by $\omega_{3}$, the spanwise vorticity at $t / T=0.8,1.0$, and 1.2 . In this fashion, we can separate the rotation from the shear via $Q$, which can be used as a measure of rotation, and then get directional information with vorticity. The vortices shed from the LEs and TEs, identified by red and blue colors, respectively, while the tip vortex is left green. The role of the tip vortex in the hovering cases studied is particularly interesting. For the case presented in Fig. 9 (delayed rotation), which shows the iso- $Q$ surfaces colored with $\omega_{3}$ vorticity, along with the spanwise distribution of lift due to pressure, the effects of the tip vortices become apparent. First, there is a low pressure region at the wingtip favorably influencing the lift (TE1). Furthermore, the tip vortex anchors the large-scale vortex pair near the tip (TE3). At midspan, however, the vortex pair has separated from the wing. This in turn drives the spanwise variation seen in the flow structures and force history.

Compared with an infinite wing, the tip vortices caused additional mass flux across the span of a low-aspect-ratio wing, which helps push the shed vortex pair, from the LEs and TEs, at midspan away from one another. Furthermore, there is a spanwise variation in effective AOA induced by the downwash (TE2), which is stronger near the tip. Overall, the tip vortices allowed the vortex pair in their neighborhood of the tip to be anchored near the wing surface, which promotes a low pressure region and enhances lift. The end result is an integrated lift value that departs from the 2 -D value considerably.

It is clear that the kinematic motions have a significant impact on tip vortex formation and the LE/TE vortex dynamics. Interestingly, for many of the kinematic motions examined, the tip vortex force enhancement could be confined to lift benefits; that is, the resulting drag did not increase proportionally.

\section{E. Region 4: Delayed Rotation, Low Angle of Attack, Low Plunging Amplitude}

Region 4 is defined by kinematics with delayed rotation, large angular amplitude (or low AOA), and shorter plunging amplitudes. Figure 10 shows the time histories of lift from the 2-D and the 3-D
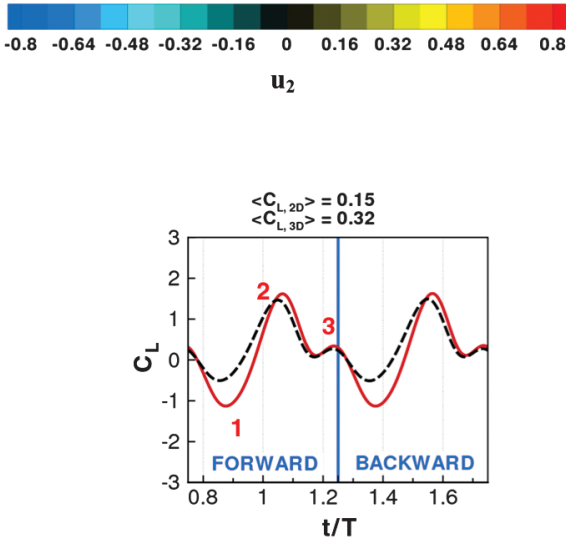

a)

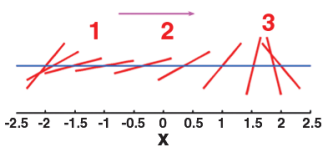

b)

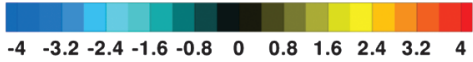

Vorticity

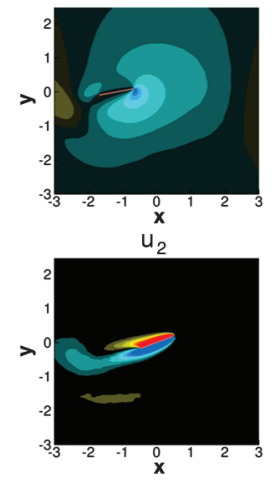

vorticity

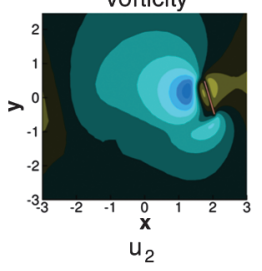

Fig. 7 Lift history and representative flow features in region 2. 
computations along with a schema for the kinematics: $2 h_{a} / c=2.0$, $\alpha_{a}=80^{\circ}$, and $\phi=60^{\circ}$ as a representative case for this region. The largest discrepancy between 2-D and 3-D is seen around $t / T=0.9$. Because the rotation is delayed, after the stroke reversal at $t / T=0.75$, the flat plate creates rotational starting vortices to increase the lift, with its first peaks around $t / T=0.9$. However, as shown in Fig. 10, in the 2-D case, the TE vortex (TEV) shed in the previous stroke interacts with the flat plate after the stroke reversal, enhancing the lift by the wake-capture mechanism $(t / T=0.9)$. On the other hand, for the 3-D case, the shed LEV and the TEV repel from each other and the path of the flat plate, such that after the stroke reversal, the wake capturing is absent. The first lift peak in the time history in Fig. 10 is then only due to the rotational effects. So the diverging behavior of the vortices observed for all delayed rotation cases, the interaction of the vortices from the LE, and the TE with the wingtip vortex play a central role as important as the 3-D effects, as described in region 3.

\section{F. Region of Similarity}

For other kinematic combinations, the integrated forces over time match reasonably: that is, the difference between the 2-D and the 3-D time-averaged lift is less than 0.1. For some cases, even the instantaneous forces agree closely: a synchronized rotation case with low AOAs is remarkably similar when examining 2-D and 3-D force histories (see Fig. 11).

Figure 11 shows the flowfields corresponding to the kinematic parameters: $2 h_{a} / c=3.0, \alpha_{a}=80^{\circ}$, and $\phi=90^{\circ}$ at $t / T=0.8,1.0$,
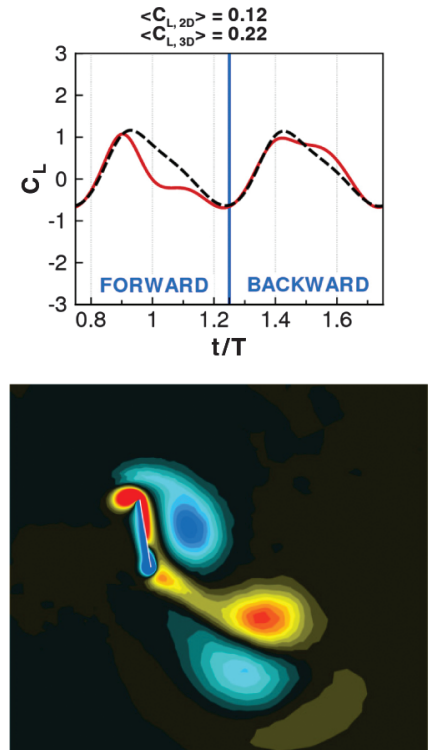

a) 2-D

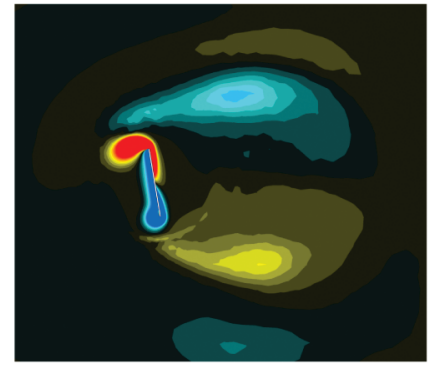

b) 3-D symmetry
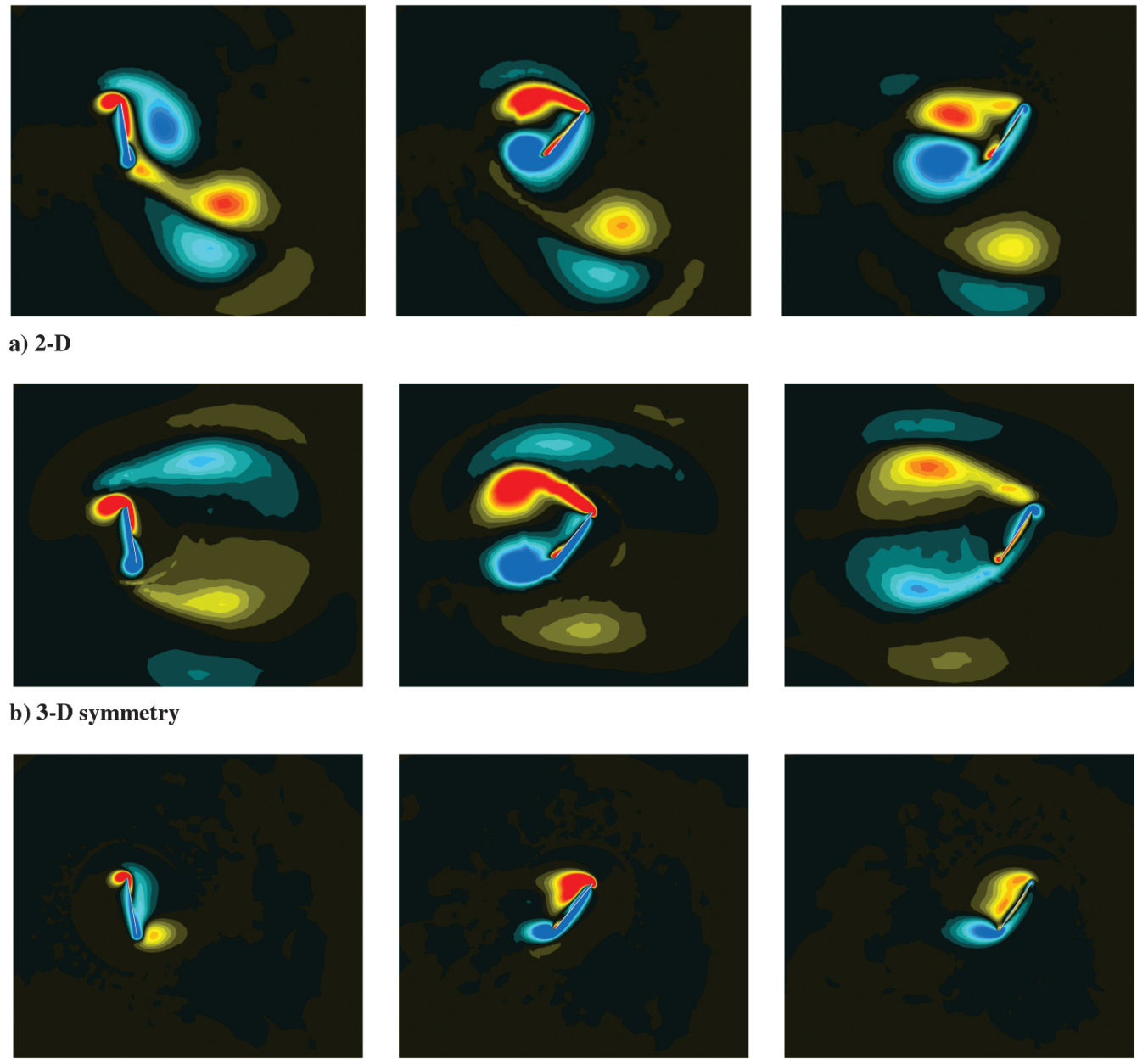

\section{Vorticity}

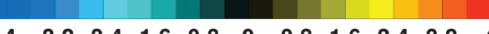

$\begin{array}{llllllllllll}-4 & -3.2 & -2.4 & -1.6 & -0.8 & 0 & 0.8 & 1.6 & 2.4 & 3.2 & 4\end{array}$

c) 3-D near tip

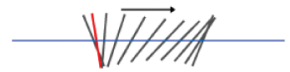

0.8

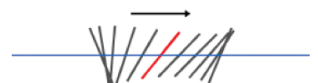

1.0
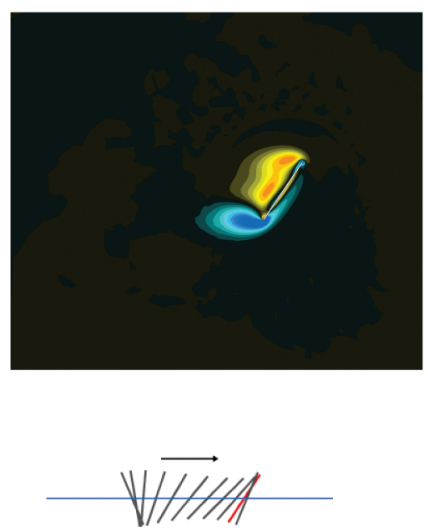

1.2

Fig. 8 Lift history and representative flow features in region 3. 

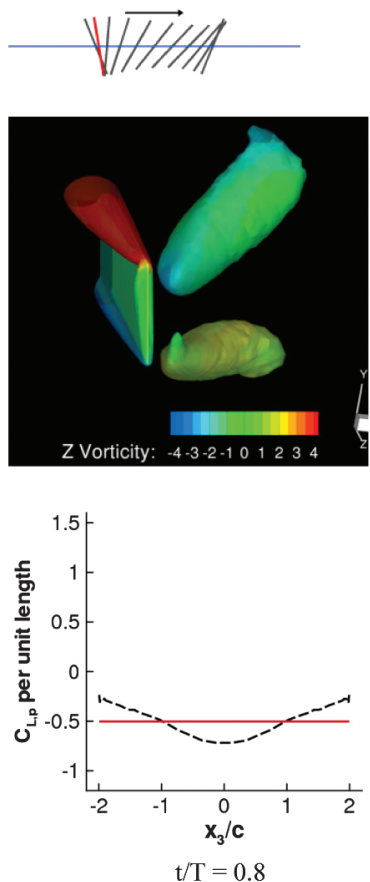
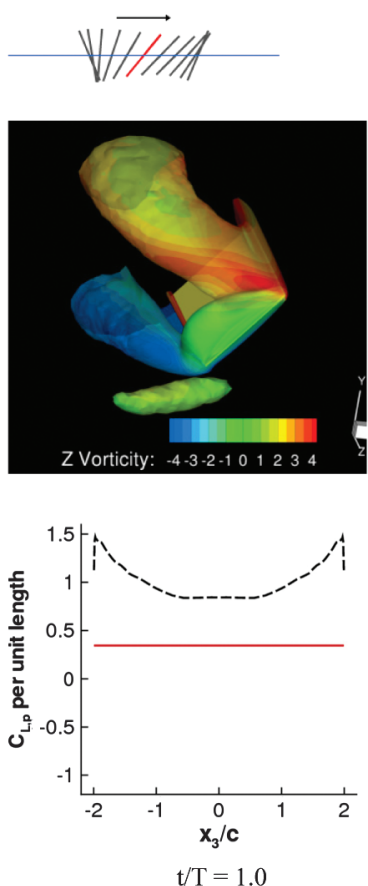
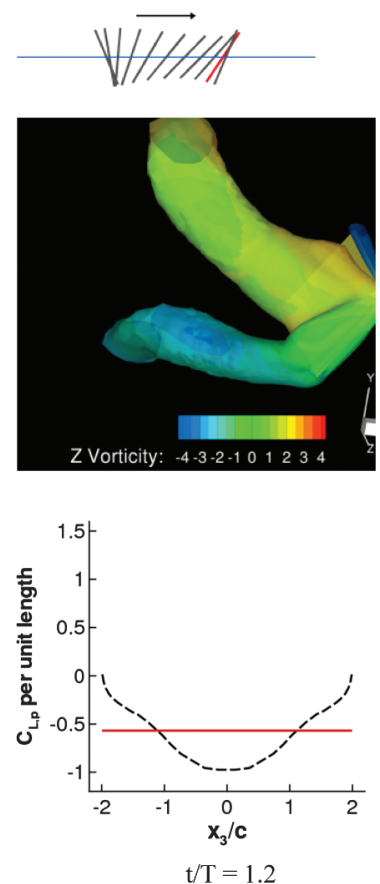

$\mathrm{t} / \mathrm{T}=1.2$

Fig. 9 The lift per unit span and 3-D vortical structures for case 1.

and 1.2. The variation along the spanwise direction is modest, making the 2-D and 3-D simulations substantially similar. The 2-D flowfield and the corresponding 3-D flow on the symmetry plane are strikingly consistent. The high angular amplitudes lead to low AOAs, and coupled with the timing of the rotation, lead to a flow that does not experience delayed stall, as the formation of the LEV is not promoted. The timing of the rotation for this example puts the flat plate at its minimum AOA (approximately $10^{\circ}$ ) at maximum translational velocity, while the translational velocity is zero when the flat plate is vertical. It is seen from the flowfield that the tip vortex as well as the LE/TE vortex formation are largely suppressed. The net effect is a fairly uniform spanwise lift distribution closely resembling the 2-D case with the same kinematics.

Neither the 2-D nor 3-D results in this case promote downward induced jet formation. As summarized in Fig. 11, the 2-D and 3-D lift coefficients of this case are similar in the instantaneous as well as the time-averaged senses. One implication illustrated is the usefulness of 2-D simulations for quantitative data on a 3-D counterpart. However, not all cases in this region display this similar instantaneous behavior, and the time-averaged lift comparison is due to integrating effects that partially cancel out. For instance, the LEV strength may diminish; however, the lift valley during the downward rotation may not be as severe in three dimensions.

\section{G. Power Requirements}

Figure 12 displays the 2-D and 3-D surrogate responses of the power required, estimated by multiplying the pressure force by the instantaneous translational velocity and integrating over a cycle, as well as their differences. Not only are all the trends quite similar, but the magnitudes also compare quite well (note the broader range of the contours seen, for the power required often yields less significant differences). This implies that certain 3-D flow features, not observable/possible in 2-D flows, for which the lift was sensitive to do not play a significant role in determining the drag for these kinematic combinations. This is an interesting consequence that warrants further study. From the instantaneous force histories (see Appendix A in [48]), the agreement in drag coefficients is very close, except at a combination of low plunging amplitudes with low angular amplitudes and low phase lags. Figure 13 measures the sensitivity of the power required to the kinematic variables. The hierarchy remains the same from 2-D to 3-D, unlike in lift.
The physical reasoning behind the observed trends in 2-D and 3-D is the same, in that the agreement is not merely a consequence of integration. During normal hovering, $\phi=90^{\circ}$, the airfoil/wing is perpendicular to the direction of motion at the ends of translation but has little translational velocity. As the phase lag is perturbed in either direction, the airfoil can have an appreciable velocity while perpendicular to the direction of motion, and whether the body is accelerating when perpendicular, as in delayed rotation cases, or decelerating, as in advanced rotation cases, has a sizable impact on the instantaneous drag that is felt (see Appendix B in [48]) and, consequently, the power required for the maneuver. Quality measures of the surrogate models are tabulated in [33]. Compared with the differences between surrogates for lift, the differences for power between 2-D and 3-D cases are smaller overall.

\section{H. Pareto Front}

In a multiobjective investigation, it is often the case that different goals are in competition regarding suitable selections of design variables. One tool used to evaluate the tradeoffs between objective functions is called the Pareto front [49]. The Pareto front consists of nondominated points and can be thought of as the set of best possibilities, as illustrated in Fig. 14 for the competing objectives of lift and power requirements in $2-\mathrm{D}$ and $3-\mathrm{D}$ as well as the design variable combinations that provide those fronts where the dashed line is provided for reference. Nondominated points can be thought of as points for which one could not improve all objective functions simultaneously. The current objectives are to maximize lift and minimize power requirement. Points on the Pareto front therefore involve those for which increases in lift are accompanied by increases in power, and vice versa. To pose all objectives as minimization expressions, any objective that is maximized, like lift, is multiplied by a negative one. Note that the Pareto front itself is very comparable between two and three dimensions. The primary differences are the peak lift values attained in 2-D flow exceeding their 3-D counterparts and the fact that the density of design variable combinations near the front is higher in the 3-D case. The paths through the design space are plotted below their respective Pareto fronts in Fig. 14. Note that the jaggedness of the path is due to the resolution of the tested points and is seen because of the fine balance in objective functions for design variables in that region. It is seen that the high lift region follows the lower bound of the angular amplitude, suggesting that future iterations should decrease the lower bound for higher lift solutions. 

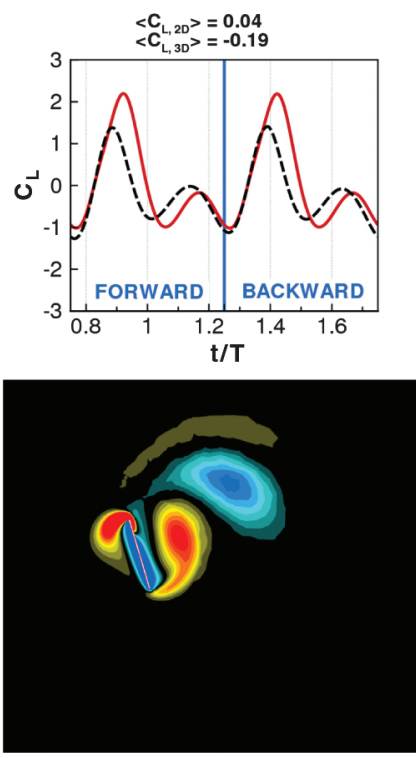

a) 2-D

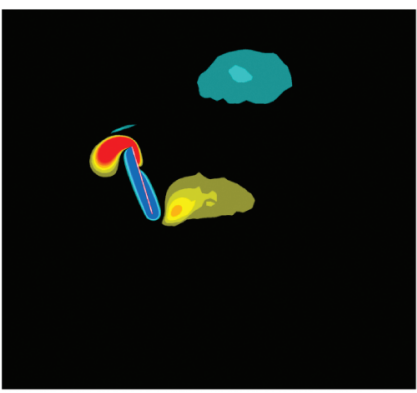

b) 3-D symmetry

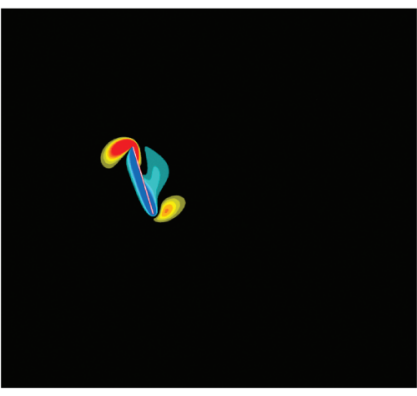

c) 3-D near tip

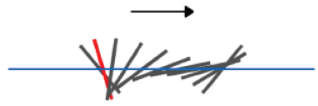

0.8
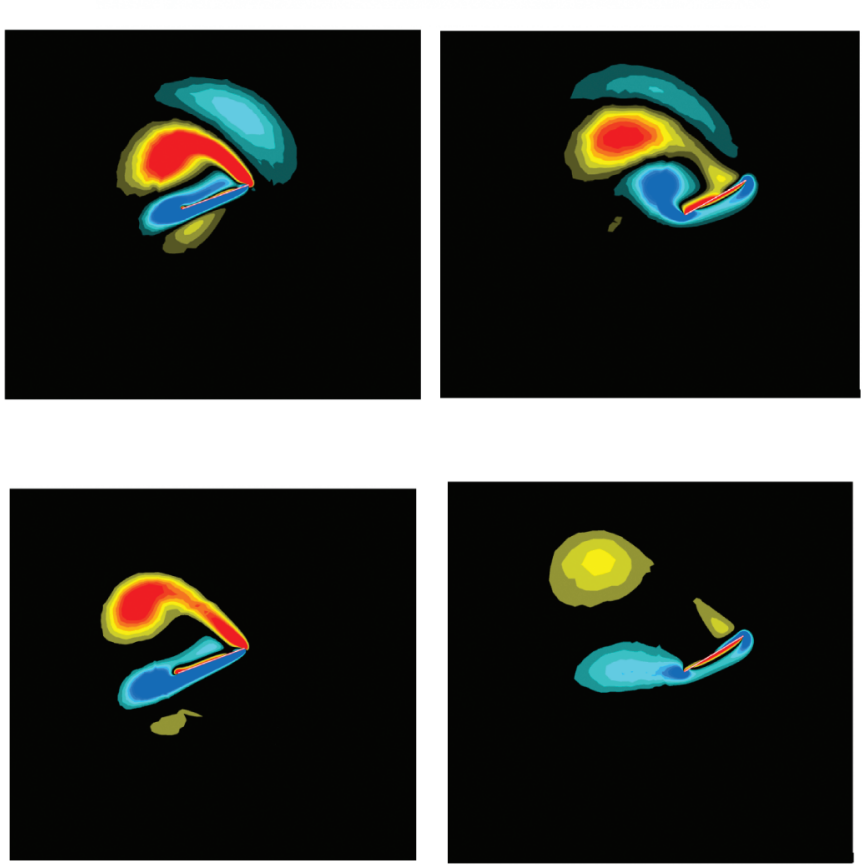

Vorticity
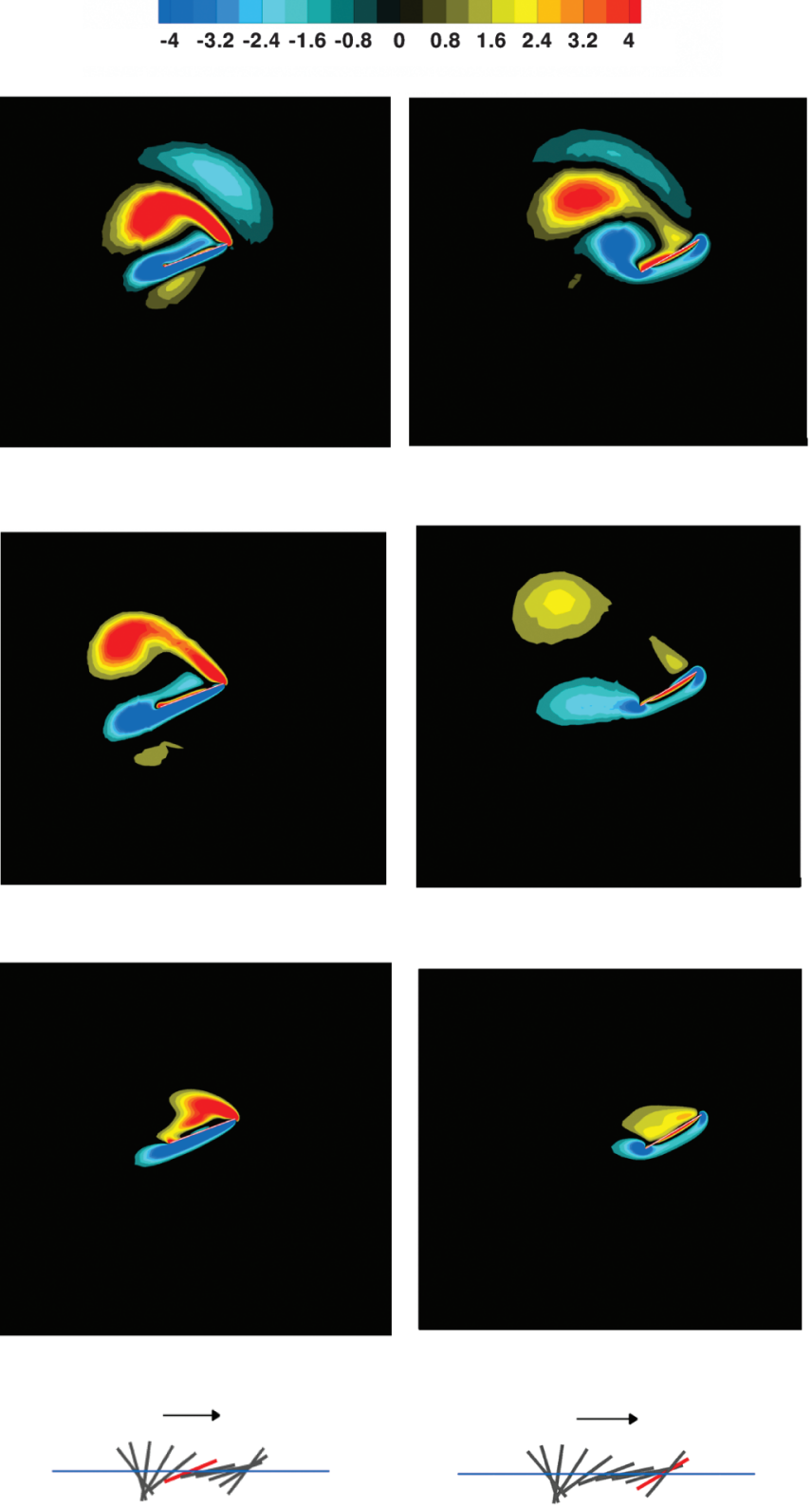

1.0

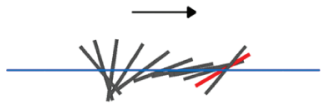

1.2

Fig. 10 Lift history and representative flow features in region 4.

Overall, the design variable combinations on the optimal front are consistent qualitatively.

As shown in Fig. 3, and a representative case illustrated in Fig. 15, the highest time-averaged lift values are obtained by a combination of advanced rotation and low angular amplitude in two and three dimensions. The general trends present (i.e., when holding two of the input variables constant and varying the last one) remained largely the same. For the power required, Fig. 14 shows that the synchronized rotation cases with high angular amplitude consume the least power for both 2-D as well as 3-D hovering. The combination of lower AOA during the midstroke when the translational velocity is at maximum and small translational velocities at the ends of the strokes when the flat plate is vertical minimizes the power. These trends not only followed similar qualitative trends between 2-D and 3 -D cases, but they also consistently quantitatively matched in magnitude.

\section{Environmental Sensitivity}

One of the main difficulties in realizing a functional MAV is its inherent sensitivity to the operating environment due to its size and weight. While the vehicle dynamics are going to be sensitive to the environmental perturbations due to the fact that the flapping frequencies are an order or two magnitude greater than the wind gusts, $\mathcal{O}(10)$ to $\mathcal{O}(100) \mathrm{Hz}$ versus wind at $\mathcal{O}(1) \mathrm{Hz}$, the aerodynamics associated with flapping wings can be pragmatically modeled 

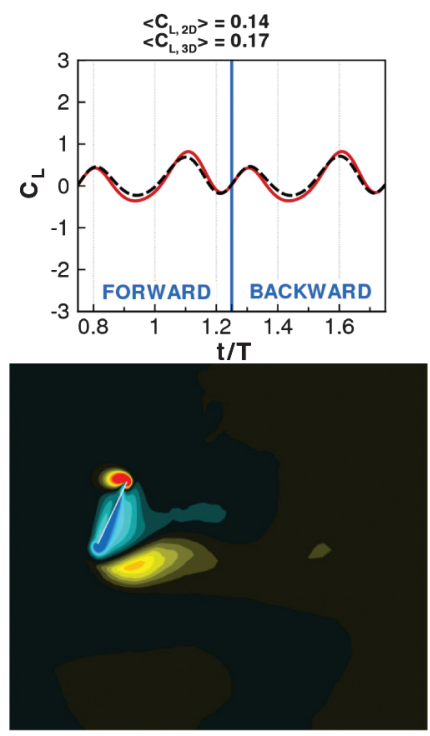

a) 2-D
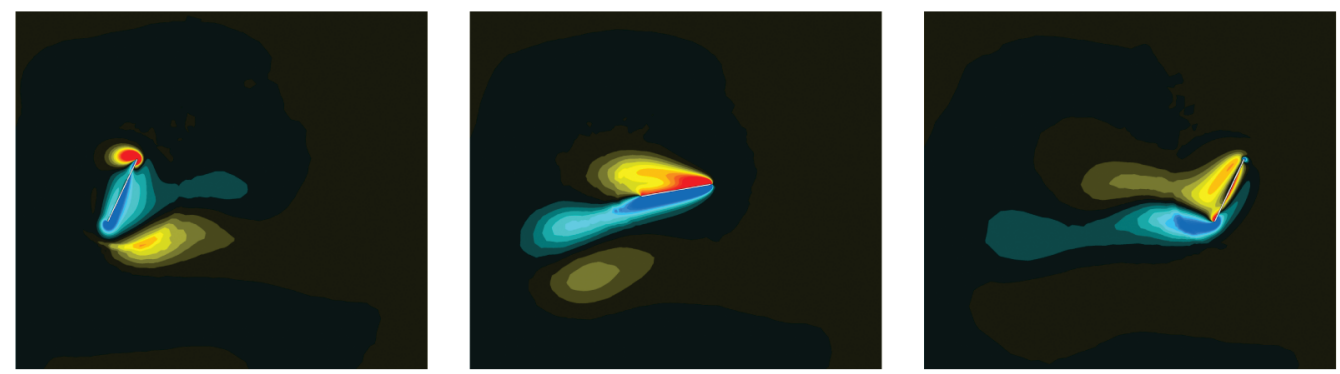

b) 3-D symmetry
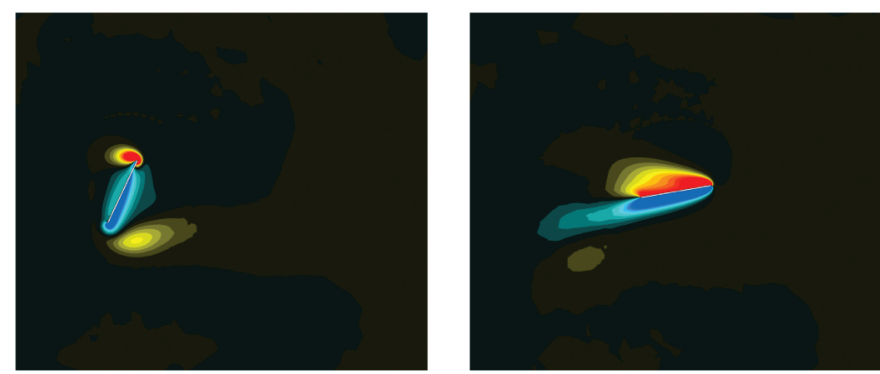

c) 3-D near tip

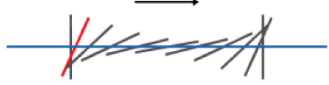

0.8

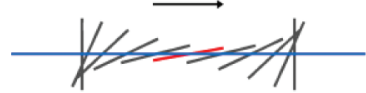

1.0
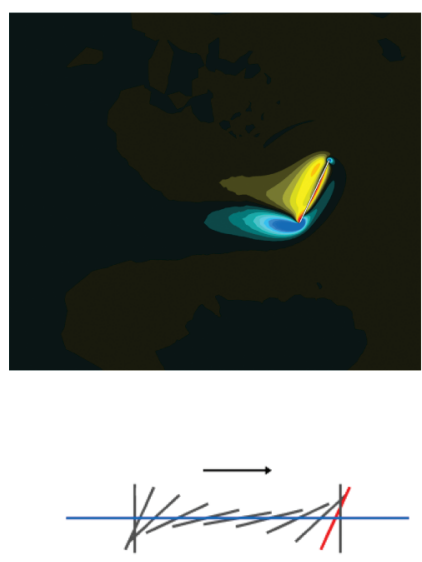

1.2 $\boldsymbol{t} / \mathrm{T}$

Fig. 11 Lift history and representative flow features in the region of similarity.

by a constant freestream. In the current study, environmental sensitivity is looked at using different kinematic schemes, freestream strengths, and freestream orientations. The three kinematic patterns chosen were those of Secs. III.B, III.D, and III.F. These patterns were chosen due to having pronounced LEVs ( $\overline{\mathrm{Sec}}$. III.B $)$, beneficial tip vortices (Sec. III.D), and negligible 2-D versus 3-D differences (Sec. III.F), respectively. The freestream strength was fixed at $20 \%$ of the maximum translational velocity of the wing. If one were comparing this to fruit flies $[\operatorname{ReO}(100)$ and wing speed $\sim 3.1 \mathrm{~m} / \mathrm{s}]$, the freestream would be approximately $0.6 \mathrm{~m} / \mathrm{s}$, a relatively light wind or mild disturbance. The directions of the freestream varied between heading down, right, or up.

The 2-D cases were much more sensitive to the freestream than their 3-D counterparts. Instantaneous lift associated with all three kinematic patterns was very sensitive to the horizontal freestream and much less sensitive to the downward heading freestream. The downward freestream generally decreased lift by suppressing vortex generation, while making the forward strokes and backstrokes more symmetric as the vortical activity was washed away from the airfoil more quickly. Overall, the general nature of the force history was kept intact. On the other hand, the upward freestream had the opposite effect. Namely, the vortex interactions were sustained for a longer period of time, as the freestream held the wake closer to the airfoil and the increased AOA also served to accentuate the unsteady aerodynamics. This upward freestream may or may not have had a significant impact on the force history, which was dependent on the kinematics. The horizontal freestream had the largest impact over the range of kinematic motions studied, sometimes more than doubling the lift felt for a freestream strength of $20 \%$ of the maximum translational velocity, a relatively tame 


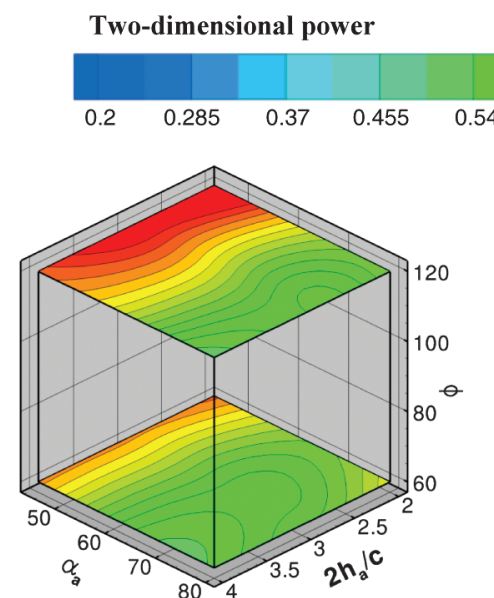

Three-dimensional power

$\begin{array}{llllll}0.625 & 0.71 & 0.795 & 0.88 & 0.965 & 1.05\end{array}$
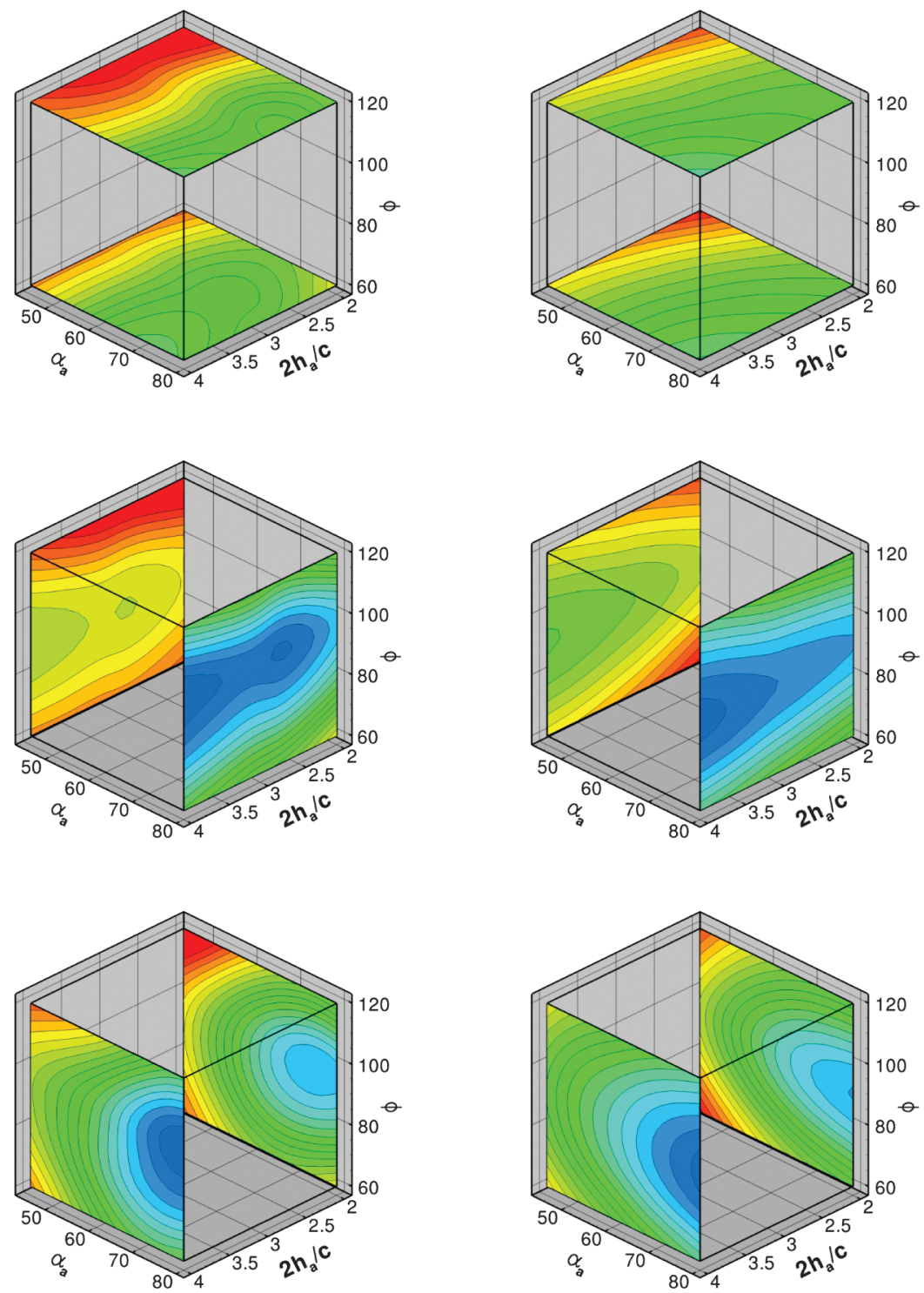
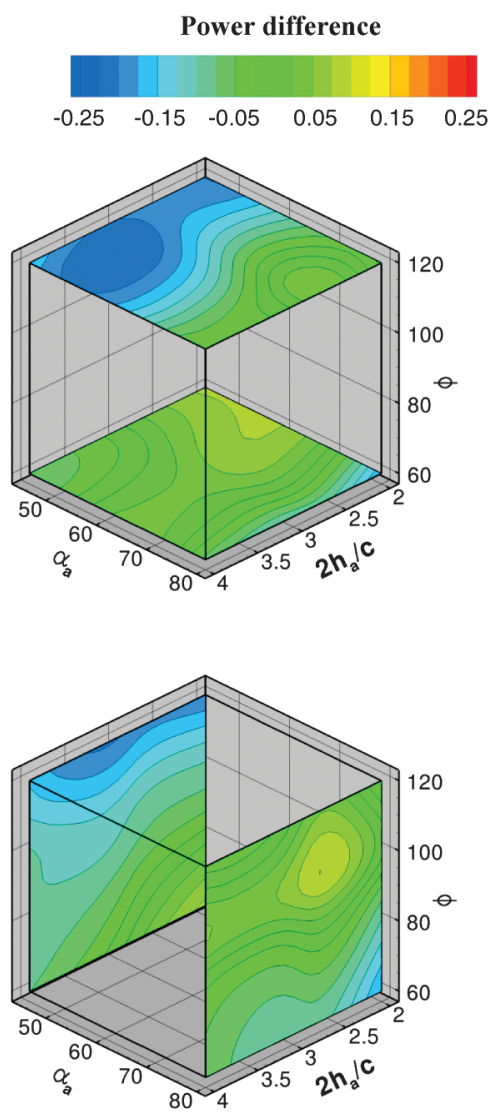

$\alpha_{a}$

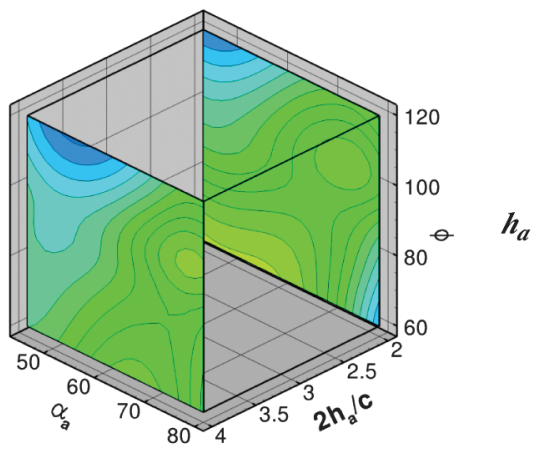

Fig. 12 Surrogate modeling results for power required: 2-D (left), 3-D (middle), and 3-D minus 2-D (right) time-averaged power requirement approximations.

environmental situation leading to a significant change in hovering performance.

Figure 16 shows the lift histories and vorticity contours illustrating the vortex formation and interactions during the LEV-dominated portion of the stroke (Figs. 16a-16c) of the wake-capture-dominated portion of the stroke (Figs. $16 \overline{\mathrm{d}-16 \mathrm{f})}$ at their respective maximal lifts for a $20 \%$ strength headwind hover scenario without freestream. The 3-D LEV-dominated portion of the stroke is highlighted with $w_{3}$ contours at two spanwise locations with a $20 \%$ freestream
(Figs. 16g-16i). Immediately apparent is the large impact on the instantaneous as well as time-averaged lift. To clarify, the lift coefficients are still normalized by the maximum translational velocity: that is, the normalization is independent of the freestream. Flowfields are plotted during the headwind portion of the stroke (backstroke) and show that the headwind case exhibits a more developed and stronger LEV, as well as stronger vorticity shedding from the TE. The increased vortical activity created by the headwind, and then interacting with the airfoil in a favorable manner,
Total Sensitivity Indices

(2-D Power)

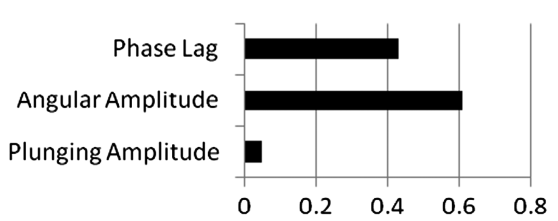

Total Sensitivity Indices (3-D Power)

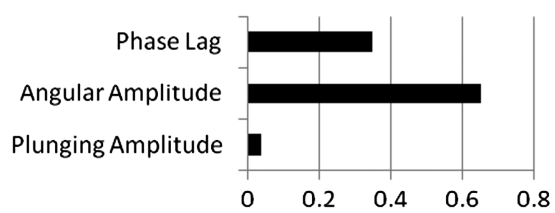

Fig. 13 GSA of power for 2-D and 3-D hovering kinematics. 

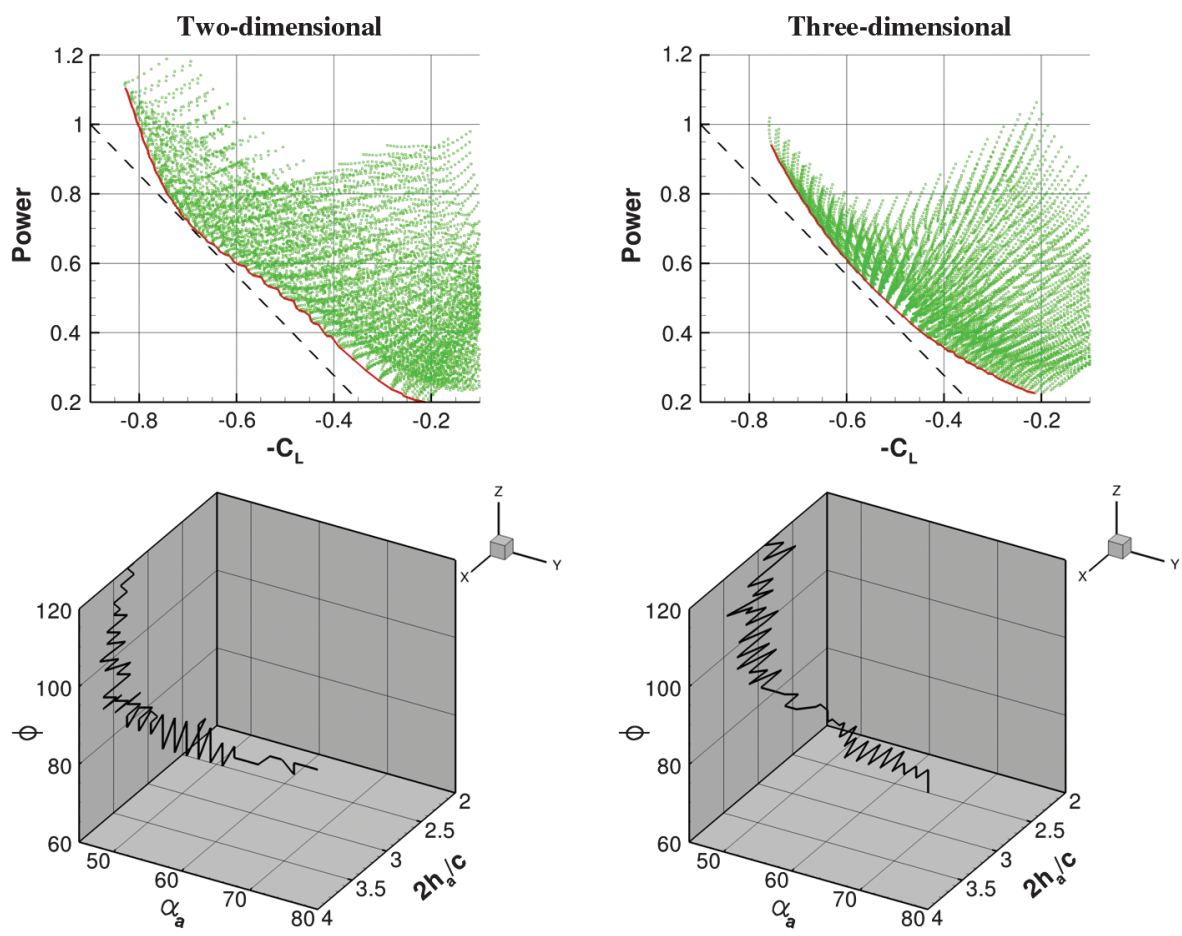

Fig. 14 Pareto fronts and its design variable combinations in 2-D (left) and 3-D (right).

qualitatively explains the increase in performance during the backstroke.

On the other hand, the significant lift peak in the presence of tailwind is due to a different lift generation mechanism. This peak occurs after stroke reversal as the airfoil interacts with the previously shed wake, or wake-capture-dominated, portion of the stroke cycle. The hover case temporarily drops off in lift (see Fig. 16d), whereas the $20 \%$ freestream case, now a tailwind, continues to increase in lift. Vorticity contours at their respective local maximums in lift (see Figs. 16e and 16f) show a few striking differences, noticeably the strength and position of the previously shed vorticity. Because of the headwind during the backstroke, stronger vortices were created. Now, on the return stroke, those vortices' strengths, in addition to their position relative to the airfoil, significantly help promote vortex growth; see Fig. 16f. This interaction, resulting in a temporary enhancement, eventually plays itself out, and a decline in lift ensues in what used to be dominated by the LEV but now amounts to slower relative translational velocity.

Looking at the 2-D force histories again (see Fig. 17a), one will see that the response of a freestream not only depends on the kinematics but also its orientation. Figure 18 illustrates the lift response to a freestream for 2-D (Figs. 18a-18c) and 3-D (Figs. 18d-18f) computations. The freestream magnitude is $20 \%$ of the maximum plunging velocity and is headed in three different directions (down, right, and up) for the three hovering kinematics (Figs. 18g-18i) investigated in the previous sections representing regions 1 and 3 and the region of similarity. The black dotted lines in Figs. 18a-18f are the lift responses of the hovering cases. For some situations, the qualitative nature of the flow does not change much over the course of the entire cycle, nor are the forces too sensitive; see the vertical freestreams in Fig. 18a or the downward freestream in Fig. 18c. On the other hand, the horizontal freestream has an appreciable impact for all of these kinematic patterns, and specific points were mentioned previously. The upward and downward freestreams do not necessarily elicit similar responses in opposite directions; highlighted in Fig. 17. This in turn brings into question the relevance of using effective $\overline{\mathrm{AO}} \mathrm{A}$ in these situations, as the nature of the force history may respond more noticeably to the upward freestream than the downward freestream; see Figs. 18a-18c, where a $20 \%$ freestream imposed from different directions changes the qualitative behavior of the resulting force history significantly.

For all of the synchronized rotation cases (which have positive AOAs at all times), a $20 \%$ downward freestream does indeed decrease the lift and follows from the suppressed vortical activity as the effective AOA is lowered. On the other hand, some cases have a much more pronounced sensitivity to the upward freestream. Figure 17a illustrates again the force histories for a $20 \%$ freestream at various orientations relative to the hover case, as well as the vorticity flowfield (Figs. 18b and 18c) for the $20 \%$ upward and downward freestream cases at a time where the difference in force history between the two is pronounced. What is seen in Fig. 17b $(20 \%$ downward freestream) and Fig. 17c (20\% upward freestream) is the increase in LEV and TEV formation as well as a more pronounced interaction with the wake as the upward freestream promotes the growth of the vortex structures and holds the wake in the vicinity for a longer period of time. The nonlinear response in lift as the freestream lowers or raises the effective AOA is a product of these factors.

The 3-D cases, on the other hand, were much less sensitive to the freestream; see Figs. 16g-16i and 18d-18f. Note, however, the scale for the force histories was chosen such that they could be directly compared with the 2-D cases, and the freestream could be quite influential. The impact was nonnegligible for a $20 \%$ strength freestream, but overall, the nature of the flow was very similar for most cases. The downward freestream once again degraded lift, and
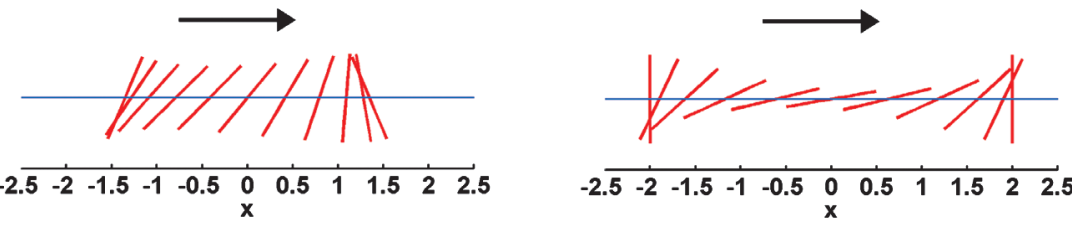

Fig. 15 Representative high lift (left; $2 h_{a} / c=2.7, \alpha_{a}=45^{\circ}$, and $\left.\phi=120^{\circ}\right)$ and low power (right; $2 h_{a} / c=4.0, \alpha_{a}=80^{\circ}$, and $\left.\phi=90^{\circ}\right)$ kinematics. 
Vorticity

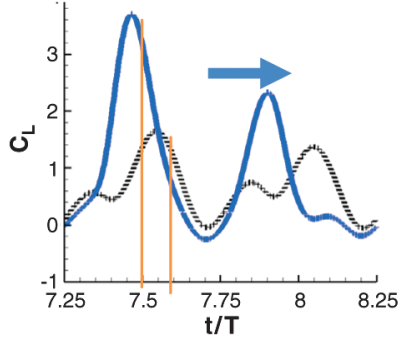

a) 2-D C $\mathrm{L}_{\mathrm{L}}$

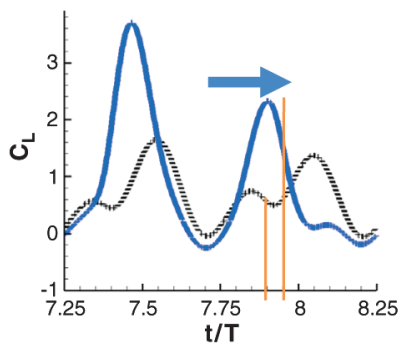

d) 2-D $\mathrm{C}_{\mathrm{L}}$

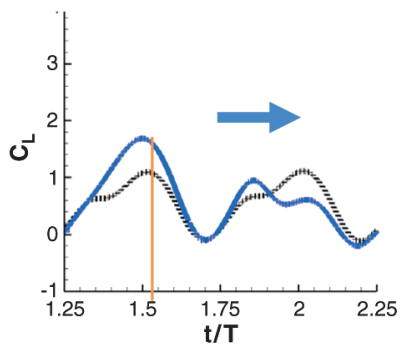

g) 3-D C $\mathrm{L}_{\mathrm{L}}$

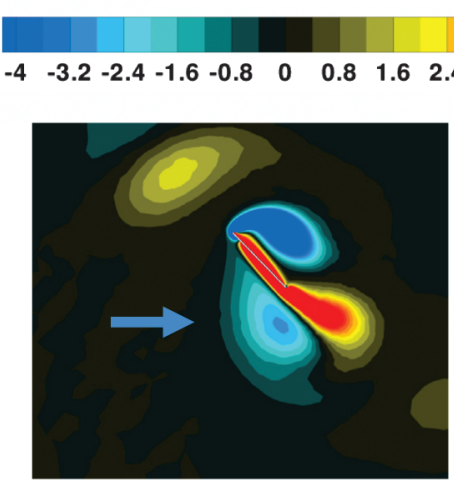

b) $20 \%$ freestream at $\mathrm{t} / \mathrm{T}=\mathbf{8 . 4 5}$

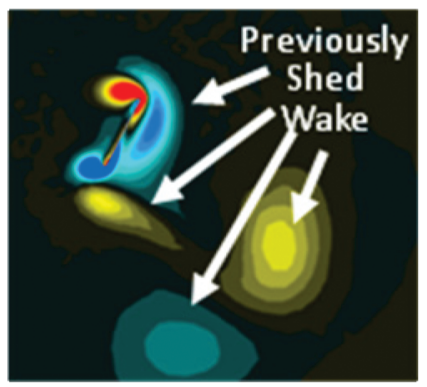

e) No freestream at $t / T=7.85$

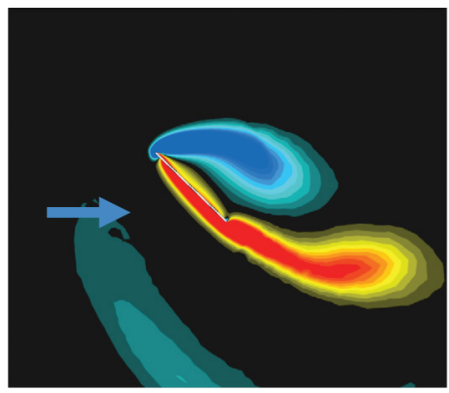

h) 3-D midspan $(\mathrm{z} / \mathrm{c}=0) \mathrm{t} / \mathrm{T}=1.520 \%$ freestream

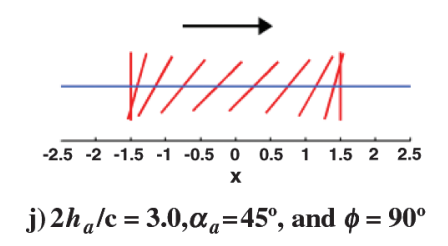

j) $2 h_{a} / \mathrm{c}=3.0, \alpha_{a}=45^{\circ}$, and $\phi=90^{\circ}$

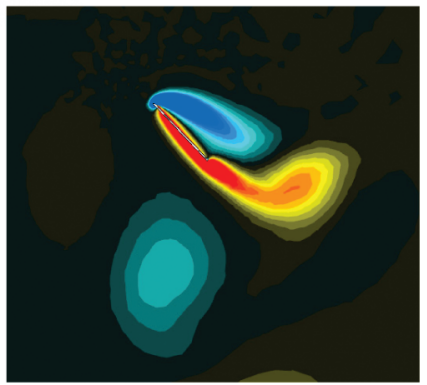

c) No freestream at $t / T=8.55$

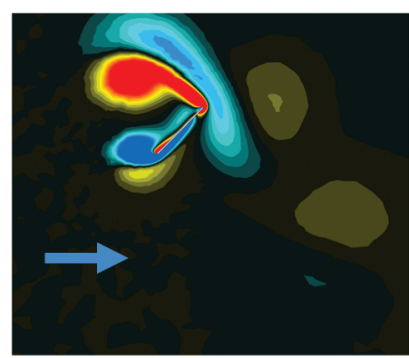

f) $20 \%$ freestream at $t / T=7.9$

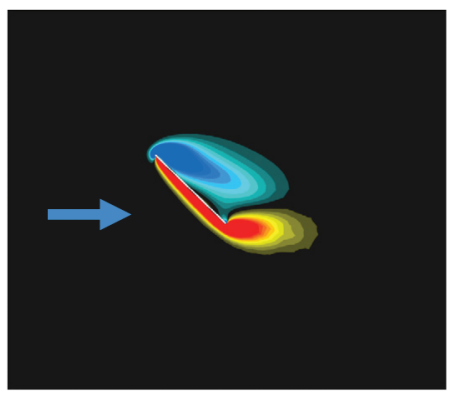

i) 3-D wingtip ( $\mathrm{z} / \mathrm{c}=1.95) \mathrm{t} / \mathrm{T}=1.520 \%$ freestream

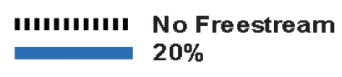

Fig. 16 Lift history and vorticity contours illustrating the vortex formation and interactions.

the upward freestream enhanced it, albeit to a lesser degree than in the 2-D cases.

Figure 19 highlights the vortex interactions at the beginning, middle, and ending of the strokes for a $20 \%$ freestream tailwind. The blue arrow in vorticity contours for $2-\mathrm{D}$ and at midspan and the wingtips for 3-D denote freestream directions. The 3-D wing is unable to generate vortices of the same magnitude as the analogous 2D counterparts. This, in turn, directly impacts the wing's benefit from LEV interactions as well subsequent interactions with the previously shed wake. The spanwise variation of vorticity exhibited shows a decrease in LEV generation from midspan to tip, and while the tip vortices are prominent, they do not make up for the weakened LEV formation and wake interaction, as experienced in 2-D. Figure 16 illustrates the vorticity flowfields during maximal lift, during the headwind, resulting in a 2-D lift (Fig. 16a) almost twice as large as its 3-D counterpart (Fig. 16g).
This discrepancy in sensitivity to freestream between 2-D and 3-D shows up across the range of kinematic motions. A limited subset of kinematic motions showed very similar force histories in the timeaveraged sense, as well as instantaneously (see Sec. III.F) when not under the influence of an external freestream. Kinematics in this region of the design space shared low AOAs across much of the flapping cycle and synchronized rotation, limiting the high angular velocities and AOAs to the end of the stroke where translational velocity was minimized. This, in turn, tended to limit vortex size, strength, formation, and influence. As the freestream is introduced (see Figs. 18c and 18f for 2-D and 3-D force histories in the presence of a $20 \%$ freestream), the response is not uniform across the span of the finite wing. The downward freestream (20\% strength) tends to suppress the vortex dynamics and, as such, the 2-D and 3-D force histories remain quite similar. The horizontal freestream, most notably during the headwind, and the upward freestream, on the other 


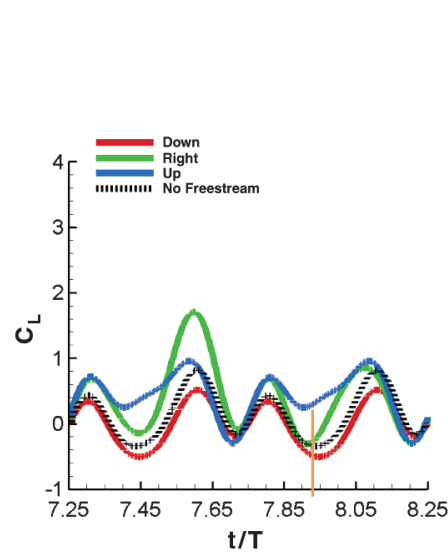

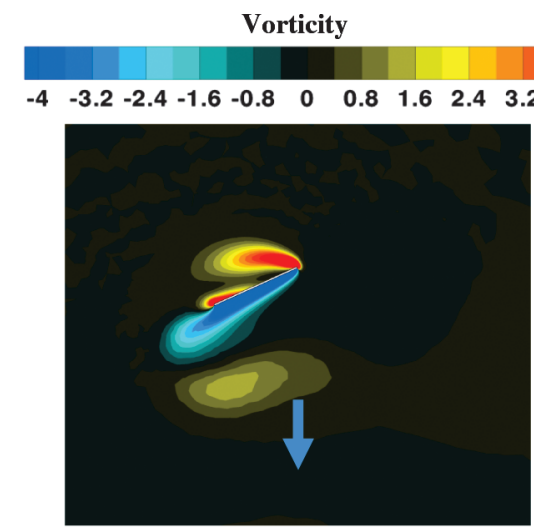

b) $20 \%$ down freestream at $\mathrm{t} / \mathrm{T}=\mathbf{7 . 9}$

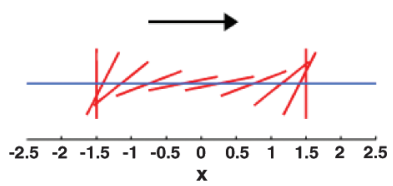

Fig. 17 Lift history and vorticity contours illustrating the vortex formation and interactions.

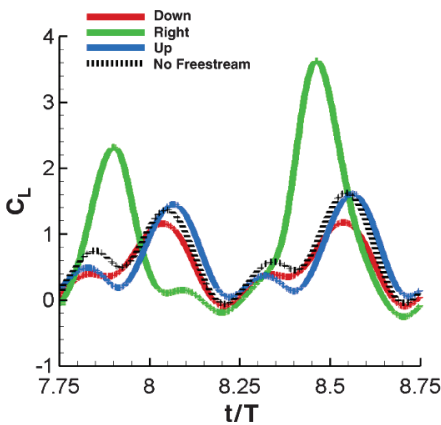

a) 2-D C $\mathrm{L}_{\mathrm{L}}$

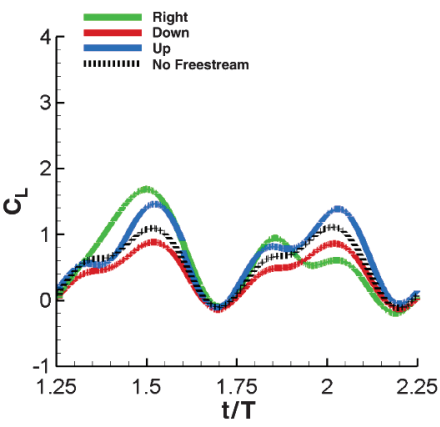

d) 3-D $\mathrm{C}_{\mathrm{L}}$

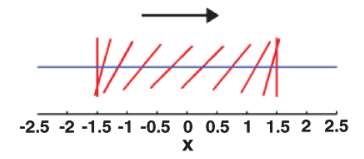

g) $2 h_{a} / \mathrm{c}=3.0, \alpha_{a}=45^{\circ}$, and $\phi=90^{\circ}$
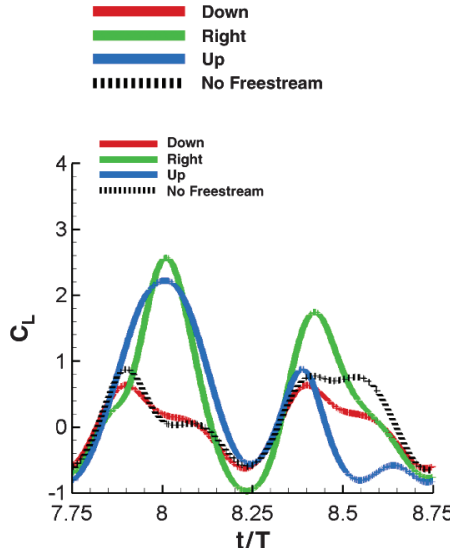

b) 2-D C

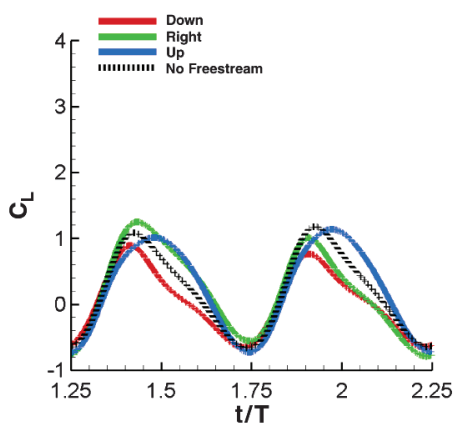

e) 3-D $C_{L}$

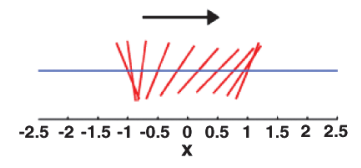

h) $2 h_{a} / c=2.0, \alpha_{a}=45^{\circ}$, and $\phi=60^{\circ}$

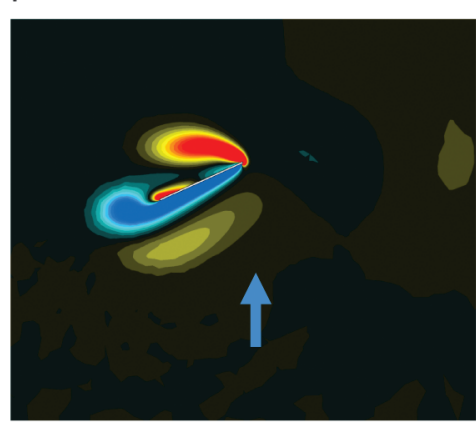

c) $20 \%$ up freestream at $\mathrm{t} / \mathrm{T}=\mathbf{7 . 9}$

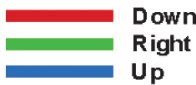

IIIIIIIII No Freestream 


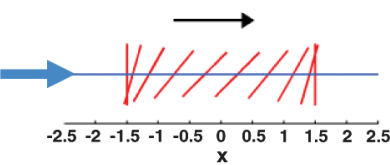

$2 h_{a} / c=3.0, \alpha_{a}=45^{\circ}$, and $\phi=90 \mathrm{~d}^{\circ}$
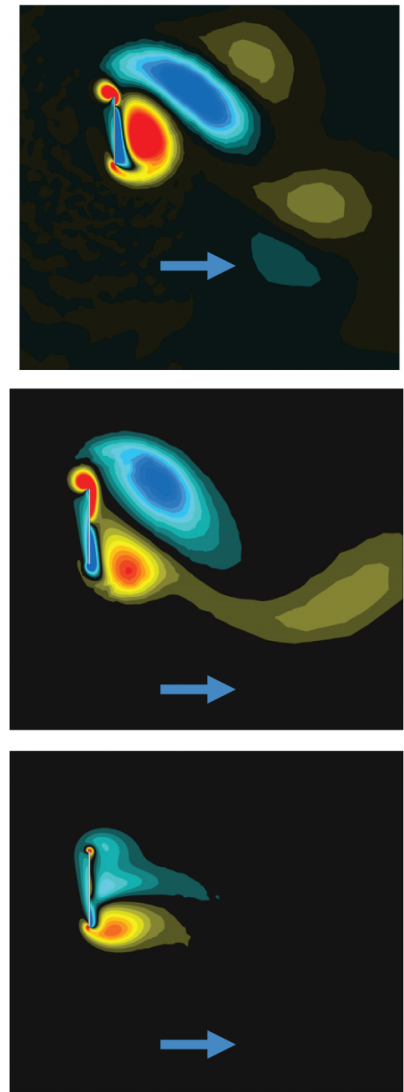

0.75

Stroke Starting
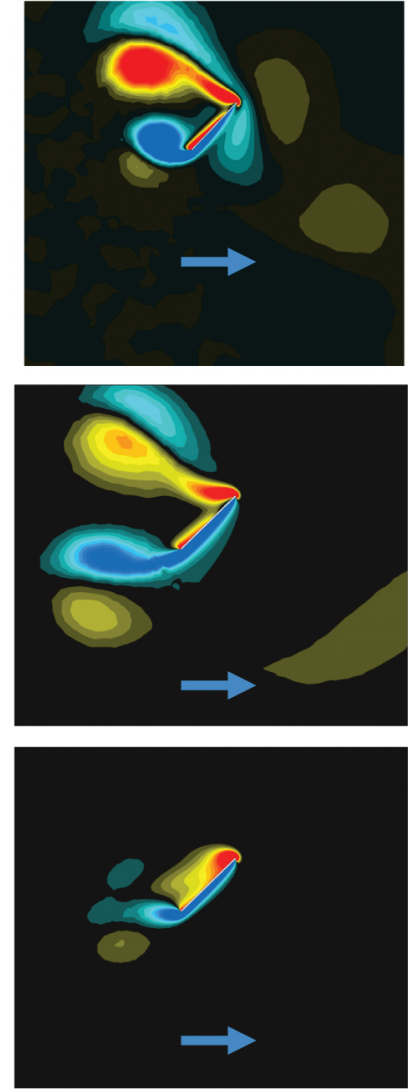

1.0

Mid-stroke
Vorticity

$\begin{array}{llllllllll}-4 & -3.2 & -2.4 & -1.6 & -0.8 & 0 & 0.8 & 1.6 & 2.4 & 3.2\end{array}$

Fig. 19 LEV generation and the subsequent wing-wake interaction under $20 \%$ freestream tailwind.

hand, show differences due to the 3-D nature of the flow; the reader is referred to [48], which includes further flowfield examinations not described here for the sake of conciseness. In the absence of a prominent freestream, we saw that the 3-D effects could accentuate the 2-D lift by creating a low pressure zone at the tip and by anchoring an LEV that would otherwise detach earlier from the wing. An interesting question, only partially addressed in the current work due to simulation resources, is to what extent the interplay between kinematic motions and the freestream strength and orientation can be manipulated.

\section{Conclusions}

In this study, hovering kinematics of 2-D and 3-D flat plates $(\mathrm{AR}=4)$ with the aid of surrogate models at a Reynolds number of 100 were examined. Regions for which large discrepancies (see Fig. 5) between the time-averaged forces in two versus three dimensions were identified with the surrogate models. Flowfield solutions from Navier-Stokes simulations were reexamined with dominated flow features highlighted for each grouping of kinematics. Regarding the previous literature, the jet experimentally seen in the studies of Freymuth [17] was seen to have a noticeable effect on the instantaneous force histories when enough care with spatial and temporal resolutions was taken. In the study of Sane and Dickinson [20], consistent conclusions were reached for obtaining optimal performance: e.g., keep fairly high AOAs for as much of the stroke as possible. There was differences, though, in the sensitivity to plunging amplitude, which was small in the current study but was relevant in the flapping about a joint method employed in their study. Furthermore, it would appear that the wake-capture portion of the cycle played a larger role, as evidenced by the force peaks they saw. In the current study, the LEV peak seems to influence the aerodynamic forces much more so than the wake-capture peak. Open questions regarding Reynolds number effects, flapping kinematics, and vortex dynamics are planned for a future study. Furthermore, new insight into the fluid physics is gained, including the effects of tip vortices.

Regarding the fluid physics and aerodynamic implications during hover, we observed the following:

1) The role of the LEV and associated delayed stall plays a dominant role in the determination of lift in both 2-D and 3-D cases. AOA, angular velocity, translational velocity, and translational acceleration all impact the formation and evolution of the LEV. For one of the delayed cases shown, the 2-D starting vortices detach, causing a weaker LEV during midstroke. In the corresponding 3-D case, the tip vortices served to anchor the LEV and, in the process, increased the time-averaged lift. The surrogate models show that this is not a general recipe for higher 3-D lift, as most delayed rotation cases at high AOA exhibit higher 2-D lift. In general, the advanced rotation cases, especially those with low AOA, were able to benefit from the 3-D effects, as the lift valley associated with strong downward rotation was much weaker in three dimensions.

2) The manifestation of the wake-capture mechanism in the 3-D case has been seen to change, as the behavior of the shed vortices may differ between 2-D and 3-D cases. This is illustrated by one case where the shed 2-D vortices would remain in the plane of the 
plunging motion. The 3-D counterparts are shed at angles such that they leave the plane of the plunging wing and do not collide with the wing upon its return.

3) In 2-D cases with higher AOAs (i.e., lower pitching amplitudes), a persistent downward jetlike flow feature was seen to form in the wake sustained by the influence of shed vortices. The jet was absent in the 2-D cases with the lowest AOAs, as there was also no large-scale vortex shedding. In the low-aspect-ratio 3-D case at $R e=100$, the presence and influence of an induced jet is not as clear cut. There are pockets of momentum encountered on return strokes, but there is no coherent reverse Kármán vortex street reinforcing the jet due to the more complex 3-D vortex interactions.

4) There was significant variance in the spanwise distribution of forces in the 3-D cases. Cases that suppressed the LEV and tip vortices, those with the highest angular amplitudes (and thus low AOAs) and small velocity when vertical (e.g., near synchronized hovering), appeared to have a relatively constant response along the span. In contrast, 3-D cases with prominent tip vortices exhibited significant variations along the span, which did not have a strong correlation to the instantaneous 2-D lift values experienced.

5) At the present Reynolds number, the 3-D flow physics and tip effects can augment the lift by a) the presence of a low pressure region near the tip, b) the anchoring of an otherwise shed vortex (in 2-D) near the tip, and c) weakening the pocket of downward momentum encountered on a return stroke. A competing effect is the induced downwash of tip vortex and the reduced effective AOA along the span, serving to reduce lift.

As flapping wing frequencies are typically much faster than that of environmental perturbations, gusts can be modeled as a constant freestream for many practical applications.

1) The sensitivity to the gust for selected kinematic parameters was largely determined on how much influence it had on the vortex dynamics. Headwinds with a modest $20 \%$ strength could significantly enhance the LEV generation, and this in turn provided stronger wake interactions on the return stroke. For most kinematic motions, within the ranges studied, a $20 \%$ horizontal freestream increased the 2-D lift despite half of the stroke experiencing a tailwind.

2) The 3-D cases, while still affected appreciably, were noticeably less sensitive to the gusts than their 2-D counterparts, due to the weakened vortex dynamics generally occurring in 3-D.

3) The impact of a gust was tied to its strength, orientation, and what kinematics it encountered. Those affected by the gust saw the manifestation through its role in LEV, tip vortex, and wake generation and interaction. Those kinematics with little of the aforementioned vortex dynamics saw the smallest sensitivity to the gust.

4) By changing the effective AOA on select kinematic motions with various freestream orientations and strengths, different values at the same effective AOAs were obtained. Hence, a cautionary note on the application of quasi-steady models in the hovering context is warranted.

Often as case studies in the literature are performed, or independent variables varied in a parametric study, it can become difficult to place the findings in the proper context, thus making it difficult to glean the relevant information and compare with findings of other researchers. The surrogate process more readily illuminates the global perspective of variables' impacts as well as the limitations of the findings, making identification of the applicability much more apparent. The surrogates modeling techniques provided a useful method for approximating otherwise computationally expensive simulations without sacrificing fidelity. Meanwhile, they proved an efficient method for analyzing trends seen throughout the design space and possible points of interest. Coupled with instantaneous force histories and flowfield measurements, they can provide insight to the complex interplay of the physical mechanisms involved. The surrogate modeling techniques further revealed the following:

1) The WAS agreed very well at the independent test points. While the number of independent test points was limited, the error was often less than a few percent. The maximum error, based on time-averaged lift values, that was found was less than $15 \%$. The agreement shows promise for using surrogates (namely, WASs) as a reduced-order model for complex engineering design in the MAV context.

2) The hierarchy of variable sensitivity in the time-averaged lift changed between 2-D and 3-D hover. In two dimensions, the importance was a) angular amplitude b) phase lag and c) plunging amplitude. In three dimensions, the hierarchy switches to a) phase lag b) angular amplitude and c) plunging amplitude. This is, in large part, from the tip vortices in 3-D simulations not suffering as large a penalty from the lift valley that would occur between wake capture and delayed stall. This difference in two dimensions and three dimensions is partially attributed to the reduced influence of the jet interaction in 3-D simulations.

3) Interestingly, the approximation to the power required remained largely the same between 2-D and 3-D design spaces. This implies that the unsteady effects that influence the drag can, in some contexts, be reduced down to their 2-D counterparts. Note the pressure force, and not the viscous force, was still the dominant component of the drag felt.

4) Regions for which 2-D kinematics outperformed 3-D in hover and vice versa were identified. Furthermore, the density of points near the Pareto front was higher in three dimensions, implying more design variable combinations that are close to optimal in terms of power and lift tradeoffs.

\section{Acknowledgments}

The work supported here has been supported in part by the U.S. Air Force Office of Scientific Research Multidisciplinary University Research Initiative grant and by the Michigan/U.S. Air Force Research Laboratory/Boeing Collaborative Center in Aeronautical Sciences. F. Viana and T. Goel are gratefully acknowledged for their useful suggestions and insight during discussions.

\section{References}

[1] Shyy, W., Lian, Y., Tang, J., Viieru, D., and Liu, H., Aerodynamics of Low Reynolds Number Flyers, Cambridge Univ. Press, New York, 2008, pp. 101-158.

[2] Shyy, W., Aono, H., Chimakurthi, S. K., Trizila, P., Kang, C.-K., Cesnik, C. E. S., and Liu, H., "Recent Progress in Flapping Wing Aerodynamics and Aeroelasticity," Progress in Aerospace Sciences, Vol. 46, No. 7, 2010, pp. 284-327. doi:10.1016/j.paerosci.2010.01.001

[3] Shyy, W., Berg, M., and Ljungqvist, D., "Flapping and Flexible Wings for Biological and Micro Air Vehicles," Progress in Aerospace Sciences, Vol. 35, No. 5, 1999, pp. 455-505. doi:10.1016/S0376-0421(98)00016-5

[4] Fearing, R. S., Chiang, K. H., Dickinson, M., Pick, D. L., Sitti, M., and Yan, J., "Wing Transmission for a Micromechanical Flying Insect," IEEE International Conference on Robotics and Automation, IEEE Publ., Piscataway, NJ, 2000, pp. 1509-1515.

[5] Lentink, D., Bradshaw, N., and Jongerius, S. R., "Novel Micro Aircraft Inspired by Insect Flight," Comparative Biochemistry and Physiology, Part A: Molecular and Integrative Physiology, Vol. 146, No. 4, 2007, pp. 133-134.

doi:10.1016/j.cbpa.2007.01.256

[6] Pornsin-Sirirak, T. N., Keenon, M., Lee, S. W., Tai, Y. C., Grasmeyer, J., Nassef, H., and Ho, C. M., "MEMS Wing Technology for a BatteryPowered Ornithopter," 13th IEEE International Conference on Micro Electro Mechanical Systems, IEEE Publ., Piscataway, NJ, 2000, pp. 799-804.

[7] Ellington, C. P., "Limitations on Animal Flight Performance," Journal of Experimental Biology, Vol. 160, 1991, pp. 71-91.

[8] Spedding, G. R., and Lissaman, P. B. S., "Technical Aspects of Microscale Flight Systems," Journal of Avian Biology, Vol. 29, No. 4, 1998, pp. 458-468.

[9] Ellington, C. P., "The Novel Aerodynamics of Insect Flight: Applications to Micro-Air Vehicles," Journal of Experimental Biology, Vol. 202, No. 23, 1999, pp. 3439-3448.

[10] Norberg, U. M. L., "Structure, Form, and Function of Flight in Engineering and the Living World," Journal of Morphology, Vol. 252, No. 1, 2002, pp. 52-81.

doi:10.1002/jmor.10013

[11] Rozhdestvensky, K. V., and Ryzhov, V. A., "Aerohydrodynamics of 
Flapping-Wing Propulsors," Progress in Aerospace Sciences, Vol. 39 No. 8, 2003, pp. 585-633. doi:10.1016/S0376-0421(03)00077-0

[12] Ho, S., Nassef, H., Pornsin-Sirirak, N., Tai, Y. C., and Ho, C. M., "Unsteady Aerodynamics and Flow Control for Flapping Wing Flyers," Progress in Aerospace Sciences, Vol. 39, No. 8, 2003, pp. 635-681. doi:10.1016/j.paerosci.2003.04.001

[13] Pines, D., and Bohorquez, F., "Challenges Facing Future Micro-AirVehicle Development," Journal of Aircraft, Vol. 43, No. 2, 2006, pp. 290-305. doi: $10.2514 / 1.4922$

[14] Wang, Z. J., "Dissecting Insect Flight," Annual Review of Fluid Mechanics, Vol. 37, No. 1, 2005, pp. 183-210. doi:10.1146/annurev.fluid.36.050802.121940

[15] Ansari, S. A., Zbikowski, R., and Knowles, K., "Aerodynamic Modeling of Insect-Like Flapping Flight for Micro Air Vehicles," Progress in Aerospace Sciences, Vol. 42, No. 2, 2006, pp. 129-172. doi:10.1016/i.paerosci.2006.07.001

[16] Ellington, C. P., van den Berg, C., Willmott, A. P., and Thomas, A. L. R., "Leading-Edge Vortices in Insect Flight," Nature, Vol. 384, No. 6610, 1996, pp. 626-630. doi: $10.1038 / 384626 \mathrm{aO}$

[17] Freymuth, P., "Thrust Generation by an Airfoil in Hover Modes," Experiments in Fluids, Vol. 9, Nos. 1-2, 1990, pp. 17-24. doi:10.1007/BF00575331

[18] Taira, K., and Colonius, T., "Three-Dimensional Flows Around LowAspect-Ratio Flat-Plate Wings at Low Reynolds Numbers," Journal of Fluid Mechanics, Vol. 623, 2009, pp. 187-207. doi: $10.1017 / \mathrm{S} 0022112008005314$

[19] Fry, S. N., Sayaman, R., and Dickinson, M. H., "The Aerodynamics of Free-Flight Maneuvers in Drosophila," Science, Vol. 300, No. 5617, 2003, pp. 295-298. doi: $10.1126 /$ science. 1084059

[20] Sane, S. P., and Dickinson, M. H., "The Control of Flight Force by a Flapping Wing: Lift and Drag Production," Journal of Experimental Biology, Vol. 204, No. 15, 2001, pp. 2607-2626.

[21] Sun, M., and Tang, J., "Unsteady Aerodynamic Force Generation by a Model Fruit Fly Wing In Flapping Motion,” Journal of Experimental Biology, Vol. 205, 2002, pp. 55-70.

[22] Wang, Z. J., Birch, M. B., and Dickinson, M. H., "Unsteady Forces and Flows in Low Reynolds Number Hovering Flight: Two-Dimensional Computations vs Robotic Wing Experiments," Journal of Experimental Biology, Vol. 207, No. 3, 2004, pp. 461-474. doi:10.1242/jeb.00769

[23] Shyy, W., and Liu, H., "Flapping Wings and Aerodynamic Lift: The Role of Leading-Edge Vortices," AIAA Journal, Vol. 45, No. 12, 2007, pp. 2817-2819. doi: $10.2514 / 1.33205$

[24] Shyy, W., Trizila, P., Kang, C., and Aono, H., "Can Tip Vortices Enhance the Lift of a Flapping Wing?," AIAA Journal, Vol. 47, No. 2, 2009, pp. 289-293. doi:10.2514/1.41732

[25] Kamakoti, R., Thakur, S., Wright, J., and Shyy, W., "Validation of a New Parallel All-Speed CFD Code in a Rule-Based Framework for Multidisciplinary Applications," AIAA Paper 2006-3063, 2006.

[26] Shyy, W., "A Study of Finite Difference Approximations to SteadyState, Convection-Dominated Flow Problems," Journal of Computational Physics, Vol. 57, No. 3, 1985, pp. 415-438. doi:10.1016/0021-9991(85)90188-3

[27] Shyy, W., Computational Modeling for Fluid Flow and Interfacial Transport, Elsevier, New York, 1994, pp. 160-163.

[28] Blazek, J., Computational Fluid Dynamics: Principles and Applications, Elsevier, New York, 2001, pp. 202-208.

[29] Luke, E. A., and George, T., "Loci:A Rule-Based Framework for Parallel Multi-Disciplinary Simulation Synthesis," Journal of Functional Programming, Vol. 15, No. 3, 2005, pp. 477-502. doi:10.1017/S0956796805005514

[30] Visbal, M. R., and Gaitonde, D. V., "High-Order-Accurate Methods for Complex Unsteady Subsonic Flows," AIAA Journal, Vol. 37, No. 10, 1999, pp. 1231-1239. doi:10.2514/2.591

[31] Visbal, M. R., and Gaitonde, D. V., "On the Use of Higher-Order FiniteDifference schemes on Curvilinear and Deforming Meshes," Journal of Computational Physics, Vol. 181, No. 1, 2002, pp. 155-185. doi:10.1006/jeph.2002.7117

[32] Trizila, P., Kang, C., Visbal, M. R., and Shyy, W., "Unsteady Fluid Physics and Surrogate Modeling of Low Reynolds Number, Flapping Airfoils," AIAA Paper 2008-3821, 2008.

[33] Trizila, P., Kang, C., Visbal, M. R., and Shyy, W., "A Surrogate Model Approach in 2-D Versus 3-D Flapping Wing Aerodynamic Analysis," AIAA Paper 2008-5914, 2008.

[34] Madsen, J. I., Shyy, W., and Haftka, R. T., "Response Surface Techniques for Diffuser Shape Optimization," AIAA Journal, Vol. 38, No. 9, 2000, pp. 1512-1518. doi:10.2514/2.1160

[35] Shyy, W., Papila, N., Vaidyanathan, R., and Tucker, P. K., "Global Design Optimization for Aerodynamics and Rocket Propulsion Components," Progress in Aerospace Sciences, Vol. 37, No. 1, 2001, pp. $59-118$. doi:10.1016/S0376-0421(01)00002-1

[36] Queipo, N., Haftka, R. T., Shyy, W., Goel, T., Vaidyanathan, R., and Tucker, P. K., "Surrogate-Based Analysis and Optimization," Progress in Aerospace Sciences, Vol. 41, No. 1, 2005, pp. 1-25. doi:10.1016/j.paerosci.2005.02.001

[37] Myers, R. H., and Montgomery, D. C., Response Surface Methodology: Process and Product Optimization Using Designed Experiments, Wiley, Hoboken, NJ, 2002, pp. 3-10.

[38] Sacks, J., Schiller, S. B., and Welch, W. J., "Design for Computer Experiments," Technometrics, Vol. 31, No. 1, 1989, pp. 41-47. doi: $10.2307 / 1270363$

[39] Cheng, B., and Titterington, D. M., "Neural Networks: a Review from a Statistical Perspective," Statistical Science, Vol. 9, No. 1, 1994, pp. $2-$ 54. doi: $10.1214 / \mathrm{ss} / 1177010638$

[40] Smola, A. J., and Scholkopf, B., "A Tutorial on Support Vector Regression," Statistics and Computing, Vol. 14, No. 3, 2004, pp. 199 222 . doi:10.1023/B:STCO.0000035301.49549.88

[41] Goel, T., Haftka, R. T., Shyy, W., and Queipo, N. V., "Ensemble of Surrogates," Structural and Multidisciplinary Optimization, Vol. 33, No. 3, 2007, pp. 199-216. doi:10.1007/s00158-006-0051-9

[42] Viana, F. A. C., Haftka, R. T., Valder, S., Butkewitsch, S., and Leal, M., "Ensemble of Surrogates: A Framework Based on Minimization of the Mean Integrated Square Error," AIAA Paper 2008-1885, 2008.

[43] Weis-Fogh, T., "Energetics of Flight in Hummingbirds and in Drosophila," Journal of Experimental Biology, Vol. 56, 1972, pp. 79104.

[44] Weis-Fogh, T., "Quick Estimates of Flight Fitness in Hovering Animals, Including Novel Mechanisms for Lift Production," Journal of Experimental Biology, Vol. 59, 1973, pp. 169-230.

[45] Ellington, C. P., "The Aerodynamics of Hovering Insect Flight, III: Kinematics," Philosophical Transactions of the Royal Society of London, Series B: Biological Sciences, Vol. 305, No. 1122, 1984, pp. $41-78$. doi:10.1098/rstb.1984.0051

[46] Anderson, J. D., Fundamentals of Aerodynamics, 3rd ed., McGrawHill, New York, 2001, pp. 351-417.

[47] Hunt, J. C. R., Wray, A. A., and Moin, P., "Eddies, Streams, and Convergence Zones in Turbulent Flows," Center for Turbulence Research, Rept. CTR-S88, Stanford, CA, 1988.

[48] Trizila, P., Kang, C.-K., Aono, H., Visbal, M., and Shyy, W., "Fluid Physics and Surrogate Modeling of a Low Reynolds Number Flapping Rigid Flat Plate," AIAA Paper 2010-5081, 2010.

[49] Miettinen, K. M., Nonlinear Multiobjective Optimization, Kluwer Academic, Boston, 1999, pp. 10-14.

P. Beran Associate Editor 